\title{
Flare activity in solar active region 8421 observed by the TRACE satellite
}

\author{
F. Zuccarello ${ }^{1}$, L. Contarino ${ }^{1}$, P. Romano ${ }^{1}$, and E. R. Priest ${ }^{2}$ \\ 1 Dipartimento di Fisica e Astronomia, Cittá Universitaria, Via S. Sofia 78, 95125 Catania, Italy \\ 2 Mathematical Science Dept., St. Andrews University, St. Andrews, KY16 9SS, UK
}

Received 7 August 2000/Accepted 12 September 2002

\begin{abstract}
Due to the wide range of wavelengths examined and to the high angular and temporal resolution, TRACE allows one to carry out a spatial and temporal analysis of active regions during highly transient phenomena such as flares. This provides new input to the study of the mechanisms involved in these phenomena. We have studied 3 flares that occurred in AR 8421 between 29 and 30 December 1998 by comparing white light, $1600 \AA$, and $171 \AA$ images obtained by TRACE with BBSO $\mathrm{H}_{\alpha}$ images, Mitaka magnetograms and Yohkoh hard X-ray data. The flares, characterized by sudden intensity enhancements in EUV loops and by moss brightenings, have been interpreted in the framework of the two canonical flare models: i.e. simple loop and two ribbon flares. Our analysis has shown that flare No. 1 may be interpreted as a two-ribbon flare triggered by reconnection between a sheared arcade and a new emerging flux tube. The analysis of flare No. 2 strongly supports the model of two-ribbon flares characterized by reconnection occurring at higher and higher levels as time proceeds. Finally, the analysis of flare No. 3 has given the opportunity to relate moss brightening with a probable process of chromospheric evaporation.
\end{abstract}

Key words. Sun: activity - Sun: flares

\section{Introduction}

Several authors have suggested that flares $\left(T_{\mathrm{e}} \sim 5-50 \times 10^{6} \mathrm{~K}\right.$; $l \sim 10^{8}-10^{10} \mathrm{~cm} ; N_{\mathrm{e}} \sim 10^{10}-10^{12} \mathrm{~cm}^{-3} ; E_{\mathrm{th}} \sim 10^{29}-4 \times$ $10^{32} \mathrm{erg}$ ) are often caused by an interaction between coronal loops and that the current sheet, which releases the energy, lies along a separator between the two magnetic bipoles (Machado et al. 1983; Mandrini et al. 1991; Inda-Koide et al. 1995; Hanaoka 1996; Machado et al. 1998a,b). Yohkoh observations have indicated that both in large two-ribbon flares (Tsuneta et al. 1992) and in compact flares (Masuda et al. 1994), there is evidence for reconnection. From some of these observations it has been proposed that a very high temperature region, situated above the flaring soft X-ray loops, is heated by fast shocks originating in flows from a high-lying reconnection site.

Shibata (1997) has built on previous suggestions (Sturrock 1980; Moore \& Roumeliotis 1992; Forbes \& Priest 1984) to present a unified model based on the magnetic reconnection process to also explain less energetic transients such as X-ray jets, EUV jets, X-ray plasmoid ejections and microflares $\left(T_{\mathrm{e}} \sim\right.$ $2-10 \times 10^{6} \mathrm{~K} ; l \sim 5-50 \times 10^{8} \mathrm{~cm} ; N_{\mathrm{e}} \sim 10^{9}-10^{10} \mathrm{~cm}^{-3}$; $E_{\text {th }} \sim 10^{26}-2 \times 10^{29} \mathrm{erg}$ ).

Recent observations with EUV imaging instruments such as SOHO/EIT have given further evidence for flare-like processes, named nanoflares, at the bottom of the energy scale

Send offprint requests to: F. Zuccarello, e-mail: fzucca@alpha4.ct.astro.it
$\left(T_{\mathrm{e}} \sim 1-2 \times 10^{6} \mathrm{~K} ; l \sim 2-20 \times 10^{8} \mathrm{~cm} ; N_{\mathrm{e}} \sim 2 \times 10^{8}-10^{9} \mathrm{~cm}^{-3}\right.$; $\left.E_{\text {th }} \sim 5 \times 10^{23}-5 \times 10^{26} \mathrm{erg}\right)$.

Understanding the mechanisms involved in these phenomena has great importance also in the context of the coronal heating question. Several authors (Krucker \& Benz 1998; Parnell \& Jupp 2000) have suggested that the energy content of the smallest (yet undetected) flares could be sufficiently large to account for the heating of the corona. In this regard, we recall that Parker (1988) suggested that random photospheric footpoint motions may cause shearing of the coronal magnetic field, inducing many tangential discontinuities whose dissipation may heat the corona.

Therefore, it seems that flare activity across the energy spectrum, i.e. from nanoflares to big flares, and coronal heating itself, might be explained in the framework of a reconnection model (Priest \& Forbes 2000, 2002). Nevertheless, this model needs to be developed much further so as to include the altitude versus time plots for hard and soft $\mathrm{X}$-ray and $\mathrm{H}_{\alpha}$ flare loop structures, the role of shocks in heating plasma and accelerating particles, the mechanisms of energy transport during the early phase of reconnection and the evaporation of chromospheric plasma into the post-flare loops.

In this scenario, the Transition Region And Coronal Explorer (TRACE), owing to its high angular and temporal resolution, gives an important contribution to the understanding of these and other phenomena occurring in the outer solar atmosphere. Up to now, TRACE has shown that the solar transition region and the corona are filled with loops that evolve rapidly in 
temperature and density and which may have an apparent diameter comparable to the angular resolution of the telescope (even if it is reasonably expected that the presently resolved structures may have unresolved substructures) (Golub et al. 1999; Schrijver et al. 1999).

The analysis of TRACE images has shown that flares may range in size from very small events in the quiet Sun to major flares in active regions, and that they are often associated with plasma flows. After the initial phase, compact bright flare loops appear; they are brightest at their tops and in most cases they disappear in a few tens of minutes. Other flare loops, initially present as short stub ends of loops often moving outward from the flare site with velocities of 10 to $20 \mathrm{~km} \mathrm{~s}^{-1}$, gradually appear as more complete structures that live for hours. Aschwanden et al. (1999) have shown that, during flares, loops may oscillate.

Moreover, the analysis of TRACE $171 \AA$ images has revealed the presence of a bright reticulated pattern of emission over active region plage areas, named moss (Berger et al. 1999a; Fletcher \& De Pointieu 1999; Martens et al. 1999), which consists of bright EUV emission elements on 1-3 $\times$ $10^{8} \mathrm{~cm}$ scales interspersed with dark holes of similar size (chromospheric jets or spicules visible in $\mathrm{H}_{\alpha}$ ). Moss is confined to a thin layer of thickness of the order of $10^{8} \mathrm{~cm}$ with a base height approximately $1.5-2 \times 10^{8} \mathrm{~cm}$ above the photosphere. The temperature is between $0.6-1.6 \times 10^{6} \mathrm{~K}$, the pressure is $0.7-1.7$ dyne $\mathrm{cm}^{-2}$ and the electron density is about $2-3$ times higher than that measured in nearby coronal loops (Berger et al. 1999b). Moss EUV elements interact with dark jets (spicules) visible in $\mathrm{H}_{\alpha}$ on a time scale of the order of $10 \mathrm{~s}$ while patches of moss persist for times of the order of $10 \mathrm{hr}$. The moss is dynamic on time scales of 10-30 s due to intrinsic changes in brightness. This temporal variability suggests that the energy source of these intensity changes occurs relatively locally (height $<10^{9} \mathrm{~cm}$ ) (De Pontieu et al. 1999).

Focussing on both themes, i.e. magnetic reconnection in interacting loops and the physics of moss, we have examined several TRACE images and carried out a detailed analysis of (1) loop behaviour during flare evolution, looking specifically at phenomena that may be caused by interacting loops, and (2) behaviour of the moss during flares. In this paper we describe the evolution of 3 flares observed in AR 8421 on December 29 and 30, 1998 (Zuccarello et al. 2001a).

In Sect. 2 we describe the characteristics of the TRACE telescope. In Sect. 3 we give a short description of the geometry we utilize to calculate some physical parameters. In Sect. 4 we describe flare loop models. In Sect. 5 we discuss the characteristics of AR 8421 during the period we have analyzed. In Sect. 6 we present the description of the TRACE image processing. In Sects. 7-9 we describe the evolution of the flares observed in AR 8421 and in Sect. 10 we discuss the results obtained and we draw our conclusions.

\section{Instrument characteristics and standard image correction}

TRACE is a NASA Small Explorer mission launched in April 1998 on a GSFC SMEX spacecraft in a Sun-synchronous orbit. The 30-cm aperture TRACE telescope uses three normalincidence coatings for EUV imagery and one for UV on corresponding quadrants of the primary and secondary mirrors. Interference filters isolate five UV bands. The images are coaligned and internally stabilized against spacecraft jitter. A lumigen coated $1024 \times 1024$ CCD detector collects images over an $8.5 \times 8.5$ arcmin field of view (0.5 arcsec pixels) (Tarbell et al. 1994; Handy et al. 1999).

The instrument collects images of the solar plasma with one arc second spatial resolution at the following wavelengths: $171 \AA, 195 \AA, 284 \AA, 1216 \AA, 1550-1700 \AA$ and white light (see Table 1 for details on ions responsible for the emission, bandwidth, temperature ranges, corresponding locations in the solar atmosphere and typical exposure times).

The original images taken by the TRACE telescope must be corrected for instrumental effects. The first step is to subtract an image of the dark pedestal (Analog-Digital-Converter (ADC) offset) by means of the standard Solar Software (SSW) provided by the TRACE team (Freeland \& Handy 1998). The pedestal and dark current value of the images is automatically obtained by the IDL routine TRACE_PREP in DN, where 1 DN is the basic data number unit which corresponds to an amplifier gain of 12 electrons/DN (for default amplifier A, Handy et al. 1999), and each electron corresponds to a detected photon that passes in the lumigen coating. The flat-fielding correction for bad pixels caused by dust particles on the fused silica filter in the aft filter wheel should be done only for white light images, as it is not necessary for EUV images (Aschwanden et al. 2000b).

A further correction must be made for hot pixels, caused by electrons which are not properly flushed out after the CCD readout and which produce a high readout current which remains high for some period of time. The next step is a correction for radiation spikes due to cosmic rays, which can be obtained by the Solar Software routine TRACE_PREP, which optionally replaces a spiky pixel (with an excess flux of $15 \%$ relative to the local median value) with this median value (defined by the nearest 8 neighbours in a $3 \times 3$ pixel area around the spike).

\section{Physical parameters}

We describe the procedure used to the determine the following physical parameters : emission measure $E M$, electron density $N_{\mathrm{e}}$, pressure $P$, thermal energy $E_{\mathrm{th}}$. We calculate these parameters in two different geometrical configurations: loops and moss. For the first configuration we consider the loops as having cylindrical shape, with $l$ and $r$ indicating the length and radius of the cylinder, respectively (see Fig. 1). In particular, we consider sections with $l=8$ pixels $\sim 2800 \mathrm{~km}$ and $r \sim 1$ pixel $\sim 350 \mathrm{~km}$. For the second configuration we determine physical parameters in portions of moss with cubic geometry having side $b$, where $b=8$ pixels $\sim 2800 \mathrm{~km}$.

The flux $f$ (or photon count rate) emitted by an optically thin plasma is proportional to the emission measure EM at the peak temperature of the instrumental response function. Therefore, if $(D N)_{\mathrm{av}}$ and $\operatorname{resp}_{(171)}$ represent the average Data Number (per unit of pixels and time) and the instrumental 
Table 1. TRACE temperature ranges and exposure times.

\begin{tabular}{lrcccc}
\hline \hline $\begin{array}{l}\text { Wavelength } \\
(\AA)\end{array}$ & Emission & $\begin{array}{c}\text { Bandwidth } \\
(\AA)\end{array}$ & $\begin{array}{c}\text { Temperature range } \\
(\mathrm{K})\end{array}$ & $\begin{array}{c}\text { Atmospheric } \\
\text { location }\end{array}$ & $\begin{array}{c}\text { Typical exposure time } \\
(\mathrm{s})\end{array}$ \\
\hline 5000 & White light & broad & $4.0 \times 10^{3}-6.4 \times 10^{3}$ & Photosphere & 0.006 \\
1700 & Continuum & 200 & $4.0 \times 10^{3}-1.0 \times 10^{4}$ & Chromosphere & 5 \\
1600 & UV cont, C I, Fe II & 275 & $4.0 \times 10^{3}-1.0 \times 10^{4}$ & Chromosphere & 0.5 \\
1216 & HI Ly $\alpha$ & 84 & $1.0 \times 10^{4}-3.0 \times 10^{4}$ & Chromosphere & 1 \\
1550 & C IV & 30 & $6.3 \times 10^{4}-2.5 \times 10^{5}$ & Transition Region & 14 \\
171 & Fe IX/ X & 6.4 & $1.6 \times 10^{5}-2.0 \times 10^{6}$ & Corona & 15 \\
195 & Fe XII/ XXIV & 6.5 & $5.0 \times 10^{5}-2.0 \times 10^{6}$ & Corona & 15 \\
284 & Fe XV & 10.7 & $1.25 \times 10^{6}-4.0 \times 10^{6}$ & Corona & 80 \\
\hline \multicolumn{5}{c}{}
\end{tabular}

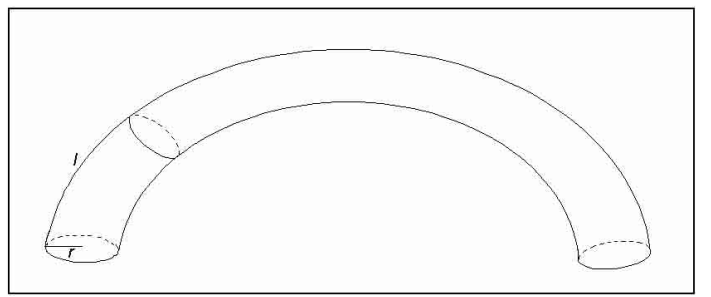

Fig. 1. Loop model with cylindrical symmetry. $l$ and $r$ are the length and radius of the cylinder, respectively.

response function of the TRACE $171 \AA$ wavelength passband, respectively, the observed emission measure is given by (Lenz et al. 1999):

$E M=\frac{(D N)_{\mathrm{av}}}{\operatorname{resp}(171)} \mathrm{cm}^{-3}$.

In the following we will adopt $\operatorname{resp}_{(171)}=9 \times$ $10^{-27} \mathrm{DN} / \mathrm{pixel} / \mathrm{s} / E M$ (obtained for $T=1.0 \times 10^{6} \mathrm{~K}$ ). Moreover, we define $E M_{1}$ as the surface emission measure, i.e. $E M_{1}=E M A$, where $A$ is the area of half the curved cylindrical surface $(A=\pi r l)$ for loops, while $A$ is the area of one cubic face $\left(A=b^{2}\right)$ when the region being analysed is the moss.

Then the volume is:

$V=\pi r^{2} l$

for the first case, and:

$V=b^{3}$

for the second one.

The electron density is given by:

$N_{\mathrm{e}}=\left(\frac{E M_{1}}{V}\right)^{\frac{1}{2}} \mathrm{~cm}^{-3}$

and the gas pressure is given by:

$P=\left(2 k_{\mathrm{B}} T_{\mathrm{e}}\right) N_{\mathrm{e}}$ dyne $\mathrm{cm}^{-2}$

where $k_{\mathrm{B}}$ is the Boltzman constant, $k_{\mathrm{B}}=10^{-15.86} \mathrm{erg} \mathrm{K}^{-1}$.

The thermal energy is:

$E_{\mathrm{th}}=\left(3 k_{\mathrm{B}} T_{\mathrm{e}} V\right) N_{\mathrm{e}} \quad$ erg.

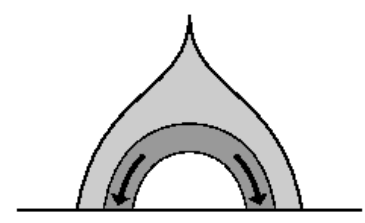

(a)

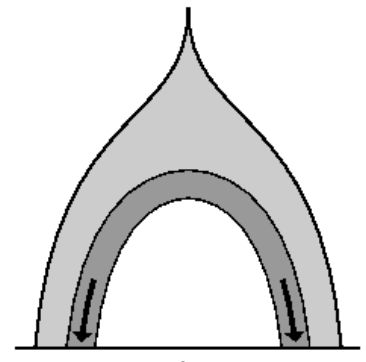

(b)
Fig. 2. Schematic drawing of a flare loop belonging to an arcade, during a two ribbon flare. a) shows a hot cusped loop created by reconnection below an erupting prominence; b) shows a higher and larger post-flare loop and plasma draining.

\section{Flare loop models}

There are two main types of flare loop that are relevant in our TRACE observations. The first is a flare loop that arises in the main phase of an eruptive two-ribbon flare. It is one of a whole arcade of loops that rises in time and joins the classical $\mathrm{H} \alpha$ flare ribbons. With TRACE one might expect to see such loops, whose location rises in height as time proceeds and the overlying reconnection point rises. Such loops are cooling from much higher temperatures. In addition at temperatures of $1 \mathrm{MK}$, one may observe the footpoints of the much hotter loops forming a pair of ribbons above the $\mathrm{H} \alpha$ ribbons either side of the polarity inversion line.

The overall scenario for the behaviour of this type of flare loop is shown in Fig. 2. First of all, reconnection below an erupting prominence creates a hot (say $3 \mathrm{MK}$ ) cusped loop (Fig. 2a) that is not visible at $171 \AA$. It then cools and retracts slightly when it may become visible in $171 \AA$. Subsequently, it cools further and drains and gradually becomes less visible at $1 \mathrm{MK}$. This whole procedure is repeated for many other loops at greater and greater altitudes as the reconnection point rises (Fig. 2b).

The second type of flare loop is produced in a non-eruptive flare by the interaction of two loops at a quasi-separatrix layer where reconnection takes place (Priest \& Demoulin 1995; Demoulin et al. 1996). First of all, reconnection takes place at some location along the loops. Heat may be dumped at this location and there may be outflows from them both of hot plasma and fast particles and conduction fronts, which 


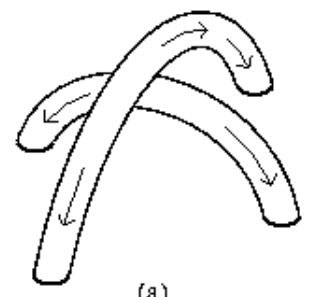

(a)

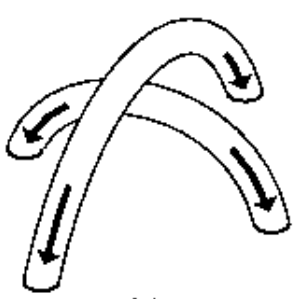

(c)

Fig. 3. Schematic drawing of two interacting loops. a) The arrows indicate heat flow and plasma and particle motions towards the loop footpoints. b) The arrows indicate chromospheric evaporation and loop filling with hot dense plasma. c) The loop cools and drains.

propagate rapidly down to the footpoints (Fig. 3a) and drive evaporation (Fig. 3b). This fills the loops with hot (several million degree) plasma that is not visible in $171 \AA$. Then the loops cool and drain, passing through the $171 \AA$ temperature regime in the process (Fig. 3c). Thus generally at $1 \mathrm{MK}$ one may see a loop only in phase (c) as it is cooling. It is also possible that a loop may be observed in phase (b) as it is heating if the loop is initially cooler than $1 \mathrm{MK}$.

If one loop is interacting with another, the source of heat in both should be at the intersection point of the loops or at their footpoints if fast particles dominate. Also a time delay of their appearance at $1 \mathrm{MK}$ in the two loops may occur if the loops are initially at different temperatures or have different lengths.

In summary: one model has filament eruption/ disappearance, rising loops, downflows and cooling loops. The other model has an interaction between two loops in which both heat up and evaporate and then both cool and drain.

These considerations lead us to pose the following questions about flaring emission seen in TRACE at $171 \AA$, so that we may determine whether or not they are examples of one of the above scenarios and what is their typical behaviour.

$Q_{1}$ : Is the emission in the form of a coronal loop? If not, is it located in a patch of moss on one side or other of the polarity inversion line, and does it represent the coronal feet of a hotter arcade of loops?

$Q_{2}$ : Is the loop cooling or heating? Is it seen first in $171 \AA$ or in the hotter $195 \AA$ ? If it is cooling, the intensity of the whole loop may decline in unison, but if it is heating the intensity may increase first at the heating source and later both sides of the heating source.

$Q_{3}$ : If the loop is heating, what is the location of the heat source? It may be identified either as the first location of an intensity rise at $1 \mathrm{MK}$ followed by a decline there and a rise in intensity either side of it. Alternatively, there may be (reconnection) outflows from the site or there may be evaporation towards that location.
Table 2. GOES events recorded in AR 8421.

\begin{tabular}{lrcccccc}
\hline \hline Date & Doy & Start & Peak & Stop & Class & Op & Location \\
\hline 23-Dec.-98 & 357 & $5: 13$ & $6: 59$ & $7: 43$ & M2.3 & - & - \\
25-Dec.-98 & 359 & $5: 31$ & $6: 34$ & $7: 27$ & M1.2 & SF & N30E66 \\
29-Dec.-98 & 363 & $5: 49$ & $6: 01$ & $6: 24$ & C2.2 & SF & N24E07 \\
29-Dec.-98 & 363 & $7: 39$ & $7: 44$ & $7: 50$ & C2.6 & SF & N26E10 \\
29-Dec.-98*(1) & 363 & $9: 23$ & $9: 41$ & $9: 53$ & C5.1 & SF & N27E13 \\
29-Dec.-98 & 363 & $20: 26$ & $20: 30$ & $20: 36$ & C2.0 & SF & N25E01 \\
30-Dec.-98*(2) & 364 & $5: 28$ & $5: 31$ & $5: 34$ & C2.9 & SF & N27W01 \\
30-Dec.-98*(2') & 364 & $5: 26$ & $5: 46$ & $6: 00$ & M1.0 & SF & N28E03 \\
30-Dec.-98 & 364 & $8: 26$ & $8: 29$ & $8: 32$ & C1.6 & SF & N27W02 \\
30-Dec.-98*(3) & 364 & $18: 04$ & $18: 10$ & $18: 15$ & C1.7 & SF & N28W06 \\
01-Jan.-99 & 1 & $12: 00$ & $12: 45$ & $13: 34$ & C3.4 & SF & N20W36 \\
03-Jan.-99 & 3 & $18: 59$ & $19: 03$ & $19: 07$ & C2.6 & SF & N21W67 \\
\hline
\end{tabular}

$Q_{4}$ : What type of flare loop is being observed? Is it a tworibbon flare loop? If so, there may have been an erupting prominence, an arcade of hot loops, $\mathrm{H} \alpha$ ribbons and an upward (apparent) motion of the loop. Or is it a compact non-eruptive prominence loop produced by reconnection at a quasi-separatrix layer? In this case most of those effects would have been absent, but is there evidence of a possible loop-loop interaction?

$Q_{5}$ : If two or more crossing loops are present, each should be analysed separately and then the question arises: are they indeed interacting or are they not? The heating locations in both loops being at the crossing point would be evidence in favour of an interaction. So would a simultaneous brightening of the two loops or a brightening of one followed after a short delay by the other.

As far as question $Q_{2}$ is concerned, it might be objected that most flares have a higher maximum temperature than what is seen in $171 \AA$, so they cool down when seen in $171 \AA$, but occasionally it is also possible to see a loop heating up to $171 \AA$ for a very weak flare.

\section{AR 8421}

We have studied 3 flares that occurred in an active region, AR 8421, characterized by quite a complex configuration and by the presence of many large and small loops (see Fig. 4). AR 8421 was seen rising at the east limb on December 22, 1998 (average latitude 26 North) and setting on January 7, 1999. During its passage across the solar disk it showed a strong flare activity: Table 2 shows the characteristics of 12 flares observed by GOES. We can see that most flares (8) occurred between 29 and 30 December, owing to a magnetic configuration characterized by three small delta spots spread along a predominantly east-west oriented neutral line. Moreover, the first three flares observed on the 29th, with a temporal cadence of $\sim 2$ hours, were characterized by an increasing energy output (from $2.2 \times 10^{-3}$ to $5.1 \times 10^{-3} \mathrm{erg} \mathrm{cm}^{-2} \mathrm{~s}^{-1}$ ).

Figure 4a shows a TRACE white light image of the entire region on December 30 at 18:29 UT, showing the high number of sunspots present in the region $(\sim 46$, as indicated in the 


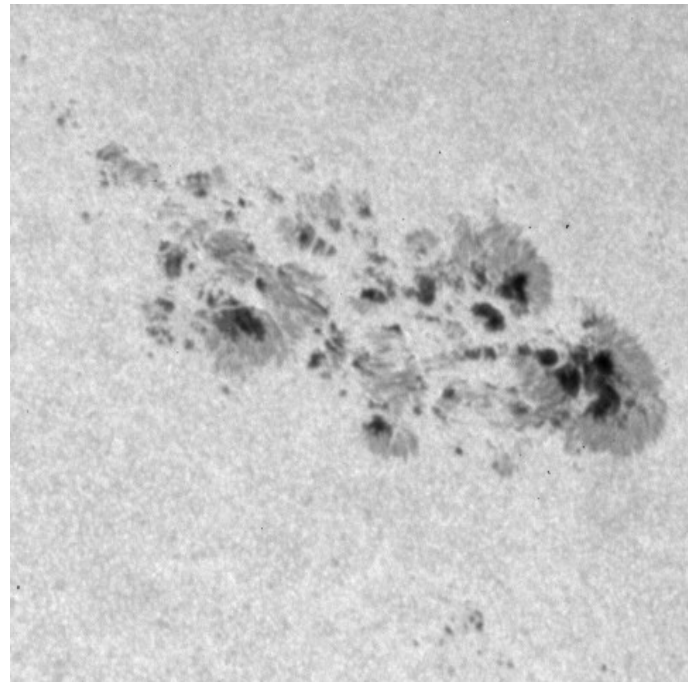

(a)

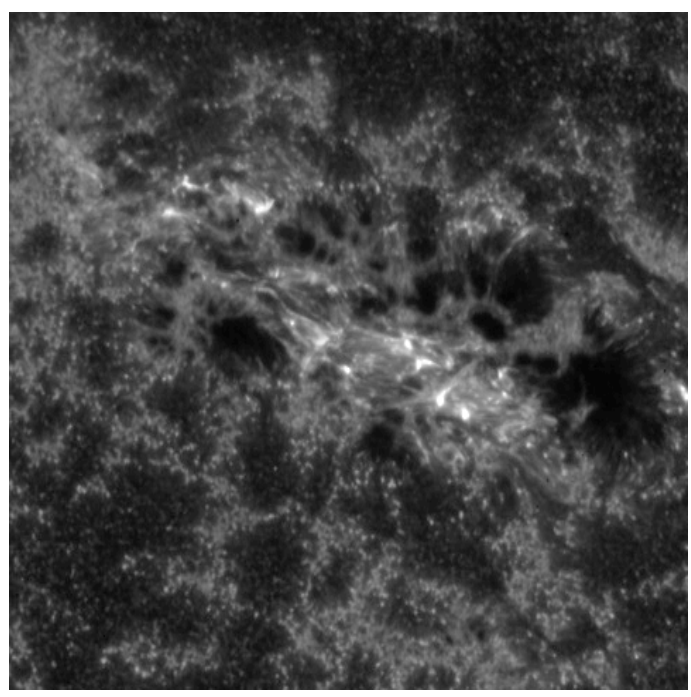

(c)

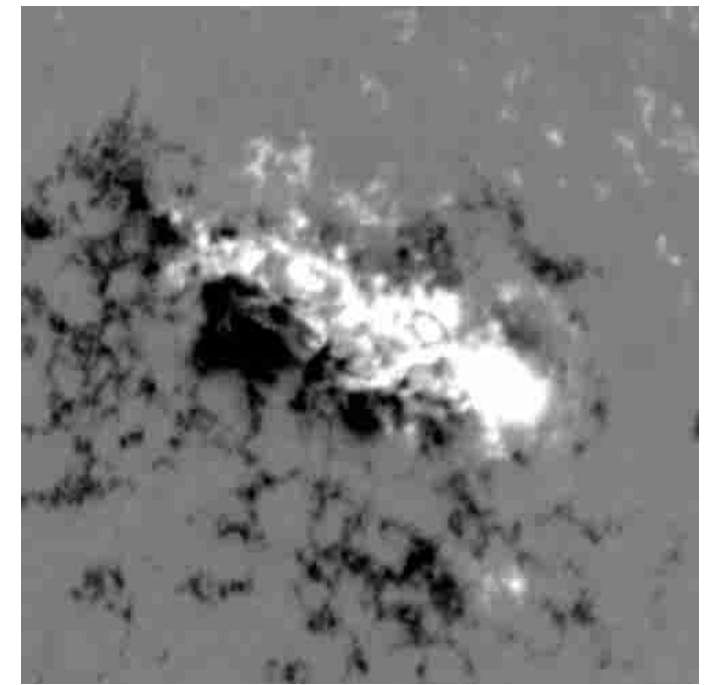

(b)

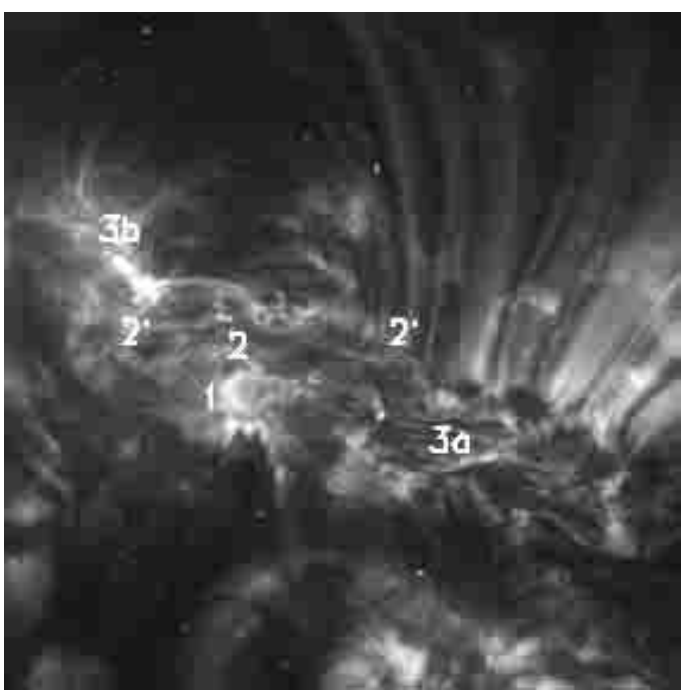

(d)

Fig. 4. Images of AR 8421 on December 30, 1998: a) TRACE WL image taken at 18:29 UT; b) BBSO magnetogram taken at 18:53 UT; c) TRACE image at $1600 \AA$ taken at 18:29 UT; d) TRACE image at 171 $\mathrm{\AA}$ taken at 18:29 UT (the numbers indicate the regions where major changes take place during the flares). The field of view in TRACE images is $\sim 175000 \times 175000 \mathrm{~km}^{2}(500 \times 500$ pixels $)$. In all the images north is on the top, west on the right.

NOAA Active Region Report); moreover, on that day AR 8421 has a total corrected area of 610 millionths of the solar hemisphere, a longitudinal extent of 13 heliographical degrees and is of $\beta \gamma \delta$ magnetic type.

In Fig. 4b we can see the BBSO magnetogram taken on December 30 at 18:53 UT, which shows an uncommon type of magnetic inversion line which runs mostly east-west instead of north-south. Moreover, some intrusions of positive polarity are visible in the left part of AR 8421.

In Figs. $4 \mathrm{c}$ and $4 \mathrm{~d}$ we can see the images taken by TRACE at 1600 (18:29 UT) and $171 \AA$ (18:29 UT), respectively, extending over an area of $\sim 175000 \times 175000 \mathrm{~km}^{2}(500 \times$ 500 pixels).

The $1600 \AA$ image in Fig. 4c clearly shows the locations of the most extended sunspots. Moreover, outside the active region, the most strongly emitting sites on the top right and bottom left have a pattern which reflects the regions of a more diffuse magnetic field in the magnetogram of Fig. $4 \mathrm{~b}$.

In the $171 \AA$ image of Fig. $4 d$ we can see that the region is characterized by the presence of both low-lying and higher loops, that there are holes in the $171 \AA$ emission which spatially coincide with the most extended sunspots visible in Fig. 4a, and that the moss is visible in different locations of the image. If we compare this image with Fig. $4 \mathrm{~b}$ showing the magnetogram, we can see that the moss coincides with the regions of more diffuse magnetic field. The numbers reported in Fig. 4d indicate the locations of the flares we have analysed.

\section{Processing the TRACE images}

We have analysed several sequences of TRACE images of AR 8421 in order to investigate the flares whose 
Table 3. Data on TRACE image sequences taken during the flares.

\begin{tabular}{|c|c|c|c|c|c|c|c|c|}
\hline $\begin{array}{l}\text { Flare number } \\
\text { and phase }\end{array}$ & $\begin{array}{c}\lambda \\
(\AA)\end{array}$ & Day & $\begin{array}{c}\text { Start time } \\
\text { UT }\end{array}$ & $\begin{array}{c}\text { End time } \\
\text { UT }\end{array}$ & $\begin{array}{l}\Delta t \\
\text { (s) }\end{array}$ & $\begin{array}{l}\text { Exptime } \\
\text { (s) }\end{array}$ & $\begin{array}{c}\text { Pointing } \\
\text { of image center }\end{array}$ & $\begin{array}{l}\text { Number } \\
\text { of images }\end{array}$ \\
\hline N. 1 Pre-flare +Flash & 171 & 1998-12-29 & $09: 25: 06$ & $09: 40: 44$ & 33 & 13.776 & $\begin{array}{ll}-277.4 & 511.9\end{array}$ & 29 \\
\hline N. 1 Main & 171 & $1998-12-29$ & 09:41:17 & 09:53:20 & 33 & 13.776 & $-277.4 \quad 511.9$ & 31 \\
\hline N. 1 Pre-flare +Flash & 1600 & $1998-12-29$ & $09: 24: 33$ & $09: 40: 50$ & 33 & 0.860 & $-277.4 \quad 511.9$ & 30 \\
\hline N. 1 Main & 1600 & $1998-12-29$ & $09: 41: 26$ & 09:59:39 & 33 & 0.860 & $-277.4 \quad 511.9$ & 34 \\
\hline N. 1 Pre-flare +Flash & WL & $1998-12-29$ & $09: 24: 37$ & $09: 40: 54$ & 33 & 0.003 & $-277.4 \quad 511.9$ & 30 \\
\hline N. 1 Main & WL & $1998-12-29$ & 09:41:30 & 09:59:43 & 33 & 0.003 & $-277.4 \quad 511.9$ & 34 \\
\hline N. 2 Pre-flare +Flash & 171 & 1998-12-30 & $05: 10: 17$ & $05: 30: 41$ & 33 & 13.776 & -2.8503 .6 & 33 \\
\hline N. 2 Main & 171 & $1998-12-30$ & $05: 31: 14$ & $05: 49: 38$ & 33 & 13.776 & -2.8503 .6 & 23 \\
\hline N. 2 Pre-flare +Flash & 1600 & $1998-12-30$ & $05: 10: 23$ & $05: 30: 50$ & 33 & 0.860 & -2.8503 .6 & 38 \\
\hline N. 2 Main & 1600 & $1998-12-30$ & $05: 31: 24$ & $05: 50: 50$ & 33 & 0.860 & -2.8503 .6 & 35 \\
\hline N. 2 Pre-flare +Flash & WL & $1998-12-30$ & 05:10:27 & $05: 30: 54$ & 33 & 0.003 & -2.8503 .6 & 35 \\
\hline N. 2 Main & WL & $1998-12-30$ & $05: 31: 27$ & 05:50:54 & 33 & 0.003 & $-2.8 \quad 503.6$ & 37 \\
\hline N. 2' Pre-flare +Flash & 171 & $1998-12-30$ & $05: 10: 17$ & $05: 45: 47$ & 33 & 13.776 & $\begin{array}{ll}-2.8 & 503.6\end{array}$ & 49 \\
\hline N. 2' Main & 171 & $1998-12-30$ & $05: 46: 20$ & $05: 49: 38$ & 33 & 13.776 & -2.8503 .6 & 7 \\
\hline N. 2' Pre-flare +Flash & 1600 & $1998-12-30$ & $05: 10: 23$ & $05: 45: 52$ & 33 & 0.860 & -2.8503 .6 & 64 \\
\hline N. 2' Main & 1600 & $1998-12-30$ & $05: 46: 26$ & $05: 50: 50$ & 33 & 0.860 & -2.8503 .6 & 9 \\
\hline N. 2' Pre-flare +Flash & WL & $1998-12-30$ & $05: 10: 27$ & $05: 45: 56$ & 33 & 0.003 & -2.8503 .6 & 63 \\
\hline N. 2' Main & WL & $1998-12-30$ & 05:46:29 & 05:50:54 & 33 & 0.003 & $-2.8 \quad 503.6$ & 9 \\
\hline N. 3 Pre-flare +Flash & 171 & $1998-12-30$ & $18: 04: 38$ & 18:09:49 & 33 & 13.776 & $138.6 \quad 483.7$ & 10 \\
\hline N. 3 Main & 171 & $1998-12-30$ & $18: 10: 22$ & $18: 29: 37$ & 33 & 13.776 & 483.7 & 34 \\
\hline N. 3 Pre-flare +Flash & 1600 & $1998-12-30$ & 18:04:45 & 18:09:54 & 33 & 0.860 & $138.6 \quad 483.7$ & 10 \\
\hline N. 3 Main & 1600 & $1998-12-30$ & $18: 10: 28$ & $18: 29: 43$ & 33 & 0.860 & 138.6 & 34 \\
\hline N. 3 Pre-flare +Flash & WL & $1998-12-30$ & 18:04:49 & 18:09:58 & 33 & 0.003 & $138.6 \quad 483.7$ & 10 \\
\hline N. 3 Main & WL & $1998-12-30$ & $18: 10: 31$ & $18: 29: 47$ & 33 & 0.003 & $138.6 \quad 483.7$ & 34 \\
\hline
\end{tabular}

characteristics are reported in Table 2.12 flares are listed in this table, but only for 3 of them could we select enough TRACE images to make a significant analysis. For most flares, in fact, there were gaps in the observations during the flash phase and the first part of the main phase. Therefore we had to restrict our analysis only to the flares occurring on December 29th and 30th which are marked by an asterisk and numbered in Table 2 .

Table 3 reports the data on the TRACE image sequences taken during each flare. In the first column we report the flare number and the flare phase; in the second the wavelength where the observations were made; in the third the day of observation; in the fourth and fifth the start and end time of the observations, respectively; in the sixth column $\Delta t$ indicates the cadence with which the images were taken by TRACE; in the seventh Exptime is the exposure time; the last two columns give the pointing of the image center from the Sun center (in arcseconds) and the number of images, respectively. All the images we have analysed were taken in full resolution mode, i.e. $768 \times 768$ pixel images with a pixel size of 0.5 arcsec and a field of view of $6.4^{\prime} \times 6.4^{\prime}$.

The procedure we have used is the following: we have analysed, for each flare, images taken during the rise phase and during the main phase separately. The raw data have been processed using the SSW routine TRACE_PREP which allows the image normalization and correction by means of the subtraction of the pedestal and dark current, as well as the correction for radiation spikes and hot pixels. For the entire 171, $1600 \AA$ and WL set of images the pedestal and dark current value is 83.9868 data numbers (DN), with a standard deviation of $\sigma_{\text {dark }}=1.51339 \mathrm{DN}$. Afterwards, the images have been coaligned as there is a slight offset $(-4.3,7.2$ pixels, see Handy et al. 1999) between the EUV channels and the WL channel caused by changes in the best focus position of the secondary mirror and by the wedge and tilt in the UV filters.

Figure 5 shows three maps of AR 8421 obtained from the $171 \AA$ images at different times. Maps (a) and (b) have a field of view of $\sim 84000 \times 84000 \mathrm{~km}^{2}(240 \times 240$ pixels $)$, while map (c) has a field of view of $\sim 140000 \times 140000 \mathrm{~km}^{2}(400 \times$ 400 pixels). These maps show the locations of the flares we have analysed, and the regions where the major changes during the flare take place are marked by a box.

In the following sections we will describe the flare evolution using $171 \AA$ images only, except for flare No. 2-2', because, despite a careful analysis of the $1600 \AA$ images, we could not obtain useful quantitative information from these data. Moreover, the analysis of WL images does not point out spot motions or other kinds of precursor phenomena before all flares, nor could we find differences in the sunspot configuration before and after the flares.

\section{Flare No. 1 observed on December 29 at 09:41 UT, C5.1}

In order to understand the kind of evolution of some loops involved in this flare, we have examined, besides the images taken during the pre-flare and flash phase (09:25-09:40 UT) 


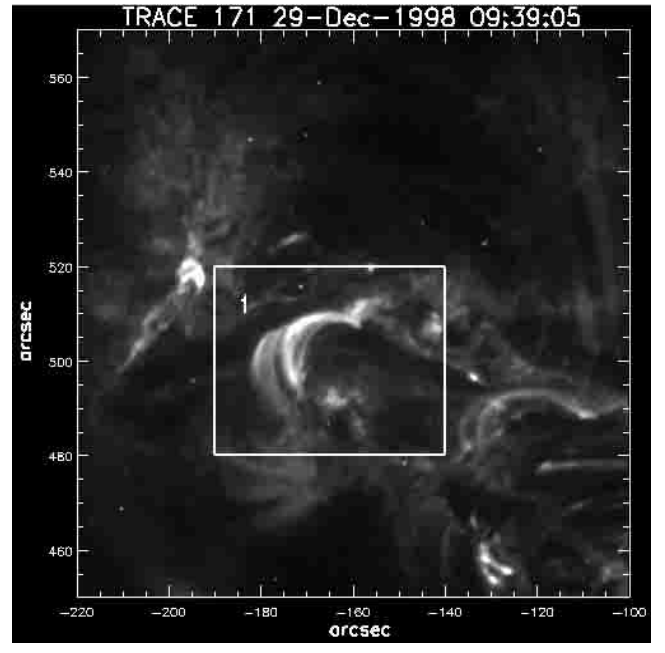

(a)

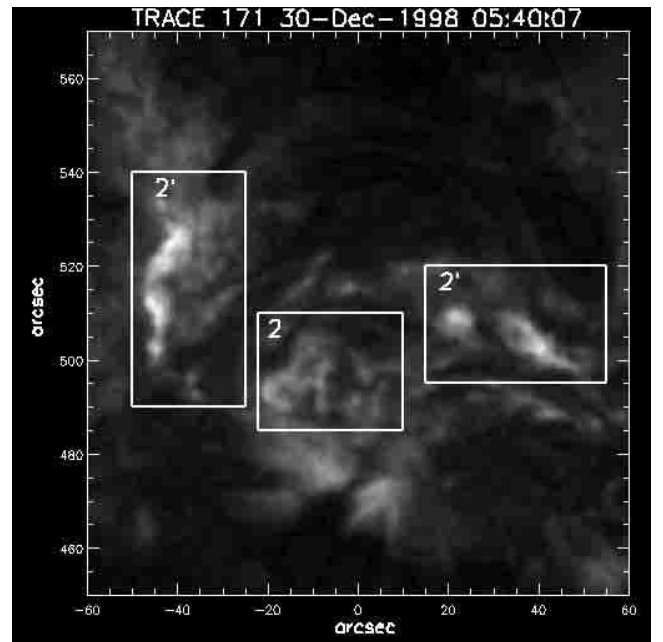

(b)

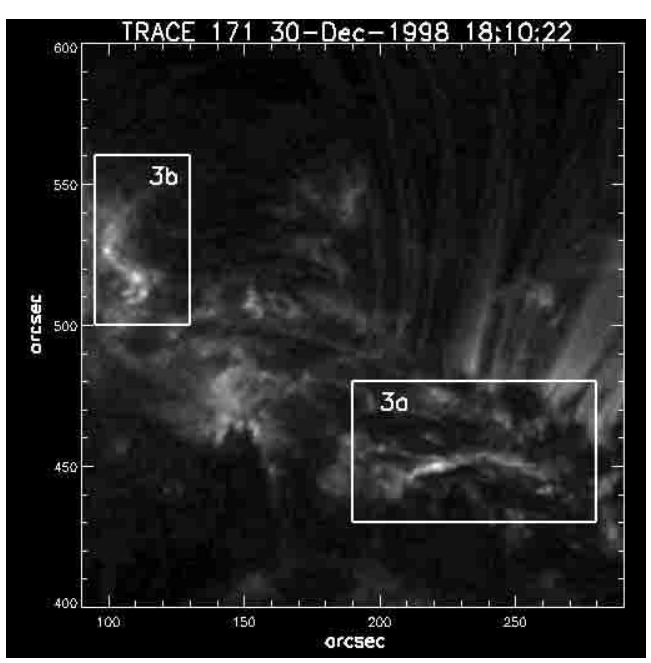

(c)

Fig. 5. Maps of AR 8421 obtained from the $171 \AA$ images taken at different times. The boxes show the regions where the major changes during the flares take place. The field of view is $\sim 84000 \times 84000 \mathrm{~km}^{2}(240 \times 240$ pixels $)$ in maps a), b), and $\sim 140000 \times 140000 \mathrm{~km}{ }^{2}(400 \times$ 400 pixels) in map c). North is on the top, west on the right. a) Map taken at 09:39:05 UT on December 29 showing the brightest system of loops involved in flare No. 1; b) map taken at 05:40:07 UT on December 30 showing the regions of maximum brightness of flare No. 2-2'; c) map taken at 18:10:22 UT on December 30 showing the positions of the brightest sites of flare No. 3 .

and during the main phase (09:41-09:53 UT), also several $171 \AA$ images taken during the preceding time interval 07:00-09:25 UT.

In Fig. 5a we can see the $171 \AA$ map of a portion of AR 8421, obtained from the image taken on December 29 at 09:39:05 UT. The heliographic coordinates of the brightest loop system $(27 \mathrm{~N}, 13 \mathrm{E})$ coincide with the coordinates given by the NOAA report.

Figure 6 shows a sequence of $171 \AA$ images of the region reported in Fig. 5a. The sequence shows two images taken a few hours before the flare $(a-b)$, two during the rise phase $(c-d)$ and two during the main phase (e-f). The white arrow in image 6 a taken at 07:55:46 UT points to the moss, in which two loops whose dimension are at the telescope resolution limit are present. These loops during the time interval between 07:55:46 and 08:02:26 UT (see Fig. 6b) show an increase both in dimension and in brightness.
Figure 6c shows the situation at 09:25:06 UT, when the region of maximum brightness coincides with the apparent contact point of the above mentioned loops. The image taken at 09:39:05 UT (see Fig. 6d), shows the situation at the flash phase, when the loops are the sites of maximum brightness in the region.

The images in Figs. $6 \mathrm{e}$ and $6 \mathrm{f}$ show the flare site during the main phase: we can see that the western loop of the most active loop system decreases its brightness and gradually disappears (Zuccarello \& Romano 1999). Beside the strong intensity increase in this loop system, we observe, during the whole time interval covered by this flare, that in the western side of the active region (see the white arrow in Fig. 6f) there are other loops with the same orientation as those we have described, which increase their intensity too. Moreover, there are patches of moss on both sides of the photospheric polarity inversion line 


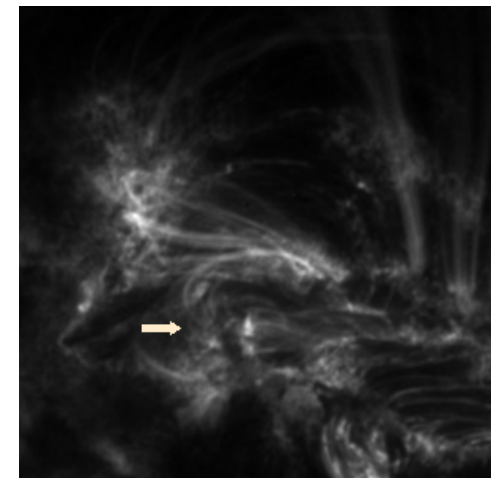

(a) 07:55:46 U.T.

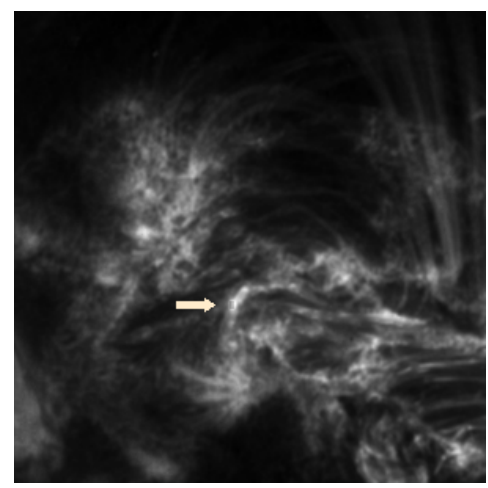

(c) 09:25:06 U.T.

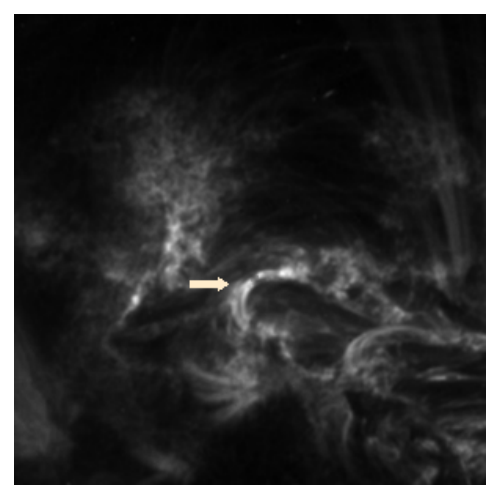

(e) 09:46:52 U.T.

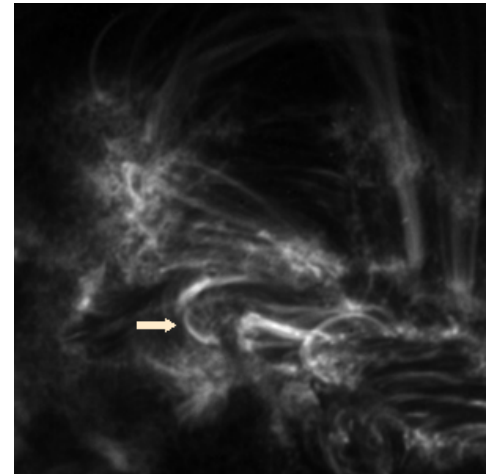

(b) 08:02:26 U.T.

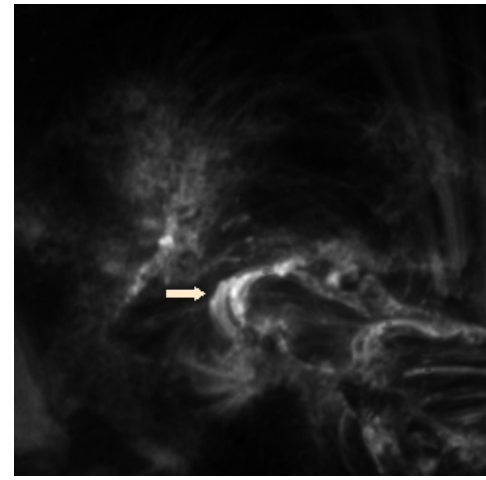

(d) 09:39:05 U.T.

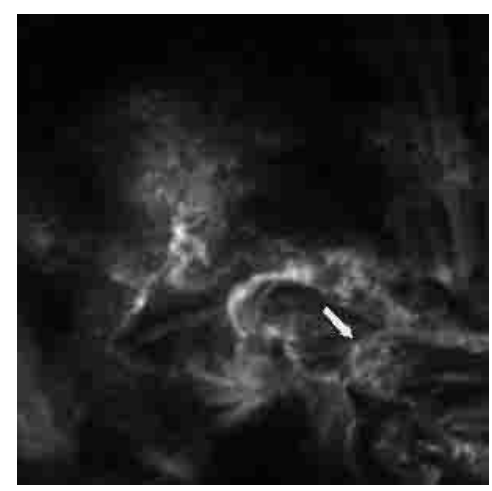

(f) 09:50:10 U.T.

Fig. 6. Sequence of $171 \AA$ images of the region reported in Fig. 5a where flare No. 1 occurred on December 29 at 09:41 UT (peak time). In each frame (except f)) the white arrow points to the brightest loop system of the site where the flare takes place. In frame f) the arrow points to the western loop system (see text). In this and all subsequent images north is on the top, west on the right. a)-b) are images taken a few hours before the flare, $\mathbf{c}$ )-d) during the rise phase and $\mathbf{e}$ )-f) during the main phase.

(hereafter referred as PIL), which increase their intensity and show a slow recessing motion from the PIL.

In order to investigate how the intensity varies along the three brightest loops, we have examined 9 frames (from 09:33:34 UT to 09:37:58 UT), in which we have divided the two eastern loops into transverse boxes of about $2 \times 4$ pixels $(700 \times 1400 \mathrm{~km})$, and the western loop into transverse boxes of about $2 \times 8$ pixels $(700 \times 2800 \mathrm{~km})$. In each box we have calculated the average Data Number (per unit of pixels and time) (Zuccarello et al. 2001b).

We mark these loops, from the eastern to the western, with the letters $a, b, c$. From the analysis of Fig. 7 we can infer that loop $b$ is the first to show an increase in its brightness (at 09:34:07 UT), while loop $a$ shows a gradual increase in its brightness in the following frames. Moreover, in loop $a$, this brightness is not uniform, but it appears to be localized at the top. Loop $c$ shows an increase in brightness with a delay of $\sim 2$ min with respect to loop $b$, but in this case the regions of maximum brightness are of greater dimensions and closer to the footpoints. Moreover, the region of apparent contact between loops $b$ and $c$ does not show an intensity increase.

Figure 8 shows the average electron density $N_{\mathrm{e}}\left(\right.$ in $\left.\mathrm{cm}^{-3}\right)$ versus time for loops $a, b$ and $c$, respectively. The peak time registered by GOES is indicated by the letters "p.t." in the 


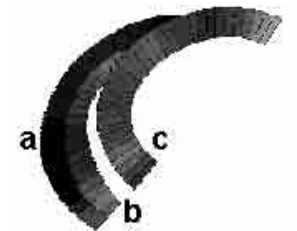

09:33:34 UT
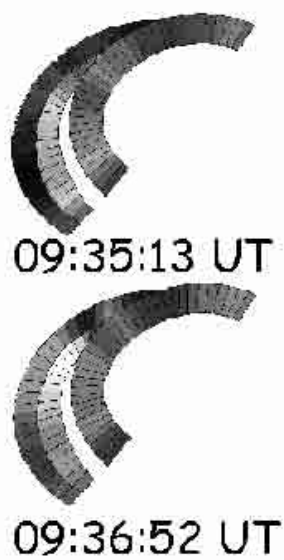

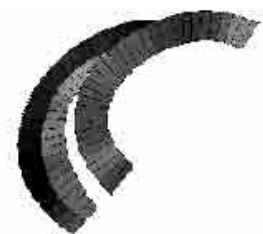

09:34:07 UT

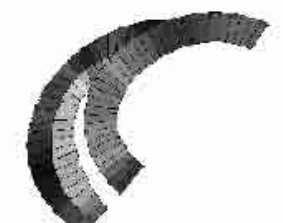

09:35:46 UT

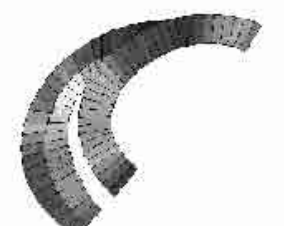

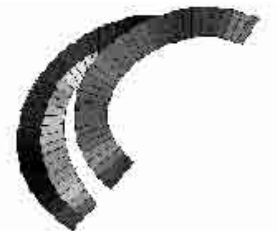

$09: 34: 40$ UT
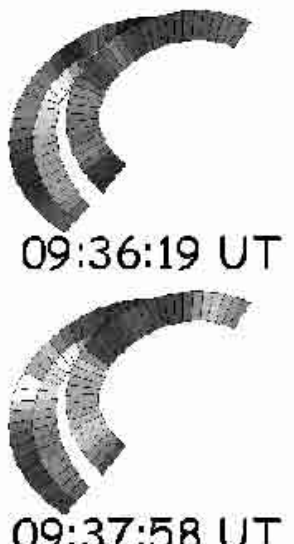

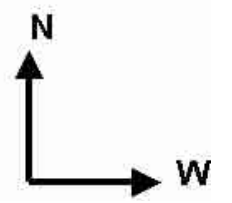

Fig. 7. The grey scale image sequence shows the average Data Number (per unit of pixels and time) along the loops involved in flare No. 1 during the pre-flare phase. The grey scale is in units of $6.5 \times \mathrm{DN} \mathrm{s}^{-1} \mathrm{pix}^{-1}$. We label these loops, from left to right, with the letters $a, b$ and $c$.

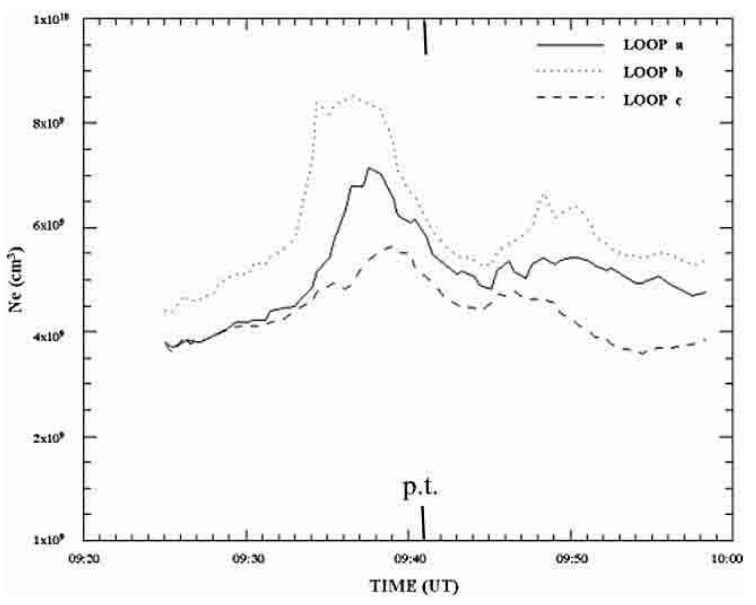

Fig. 8. Average electron density $N_{\mathrm{e}}\left(\right.$ in $^{-3}$ ) versus time in loops $a, b$ and $c$. In this graph p.t. indicates the peak time registered by GOES.

graph. We can see that the average electron density $N_{\mathrm{e}}$ of loop $b$ increases first, that this increase is the most rapid and that the maximum $N_{\mathrm{e}}$ value is the higher between the three curves. We can also notice a secondary maximum after $\sim 12 \mathrm{~min}$. The curve relating to loop $a$ indicates that the maximum is reached with a delay of $\sim 1 \mathrm{~min}$ with respect to loop $b$. Finally, the curve showing the average electron density $N_{\mathrm{e}}$ of loop $c$ indicates that this loop reaches its maximum electron density after $\sim 2 \mathrm{~min}$ with respect to loop $b$, and that there is a secondary maximum. Therefore we deduce that in each loop the density increases during the rise phase, it is in a decreasing phase at the peak time registered by GOES, and also shows a secondary maximum. Moreover, $\Delta t_{\max }$, the time interval between the first and the second maximum in each loop, is longer for the brighter loops and for those which brighten first.

In Fig. 9 we show, for each loop, the intensity $I$ (in DN/pixel/s) as a function of $s$, the coordinate along the loop, at 7 different times: curves 1-4 (solid line) refer to times before the primary maximum (deduced for each loop from Fig. 8), while curves 5-7 (dashed line) refer to times following this maximum. In each graph $s=0$ coincides with the eastern footpoint, while $s=1.0$ coincides with the western part of the loops still visible in the $171 \AA$ images. Let us examine the three curves separately.

Figure 9a refers to loop $a$ and indicates that during the ascending phase to the maximum, (solid lines), the $I$ increase takes place in two regions, one closer to the top of loop $a$ and another closer to the western footpoint; then the two peaks approach each other. Later, during the descending phase, the region of maximum brightness shifts towards the western footpoint.

In Fig. 9b we can see that the intensity in loop $b$ along the coordinate $s$ shows a gradual increase which in curves 3 and 4 shifts towards the region of apparent contact between the three loops (see Fig. 7 for a comparison). The dashed curves related to times after the maximum indicate that the region of maximum intensity is still located in the neighbourhood of the contact region.

Figure 9c, shows the intensity variations along loop $c$ at 7 different times. The intervals labelled (a) and (b) on the axis of the abscisse indicate the regions of apparent contact between loop $c$ and loops $a$ and $b$ respectively. This graph 


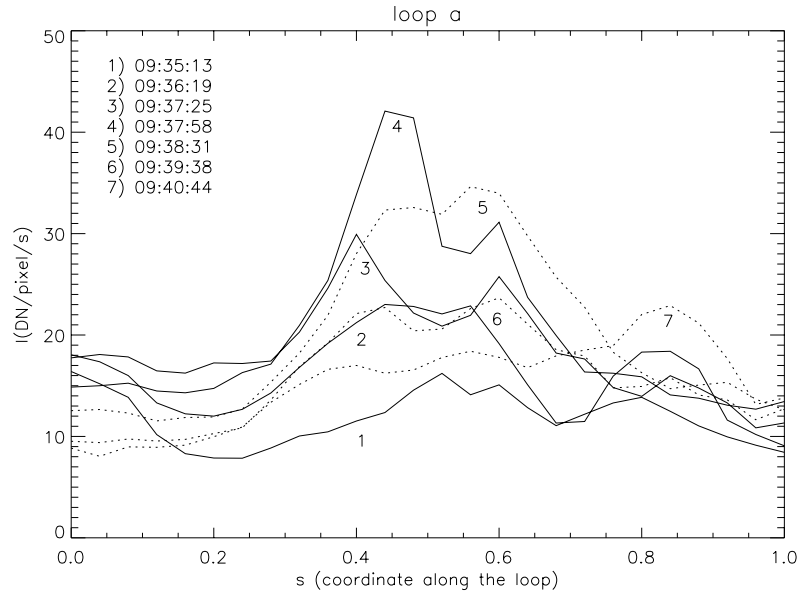

loop b

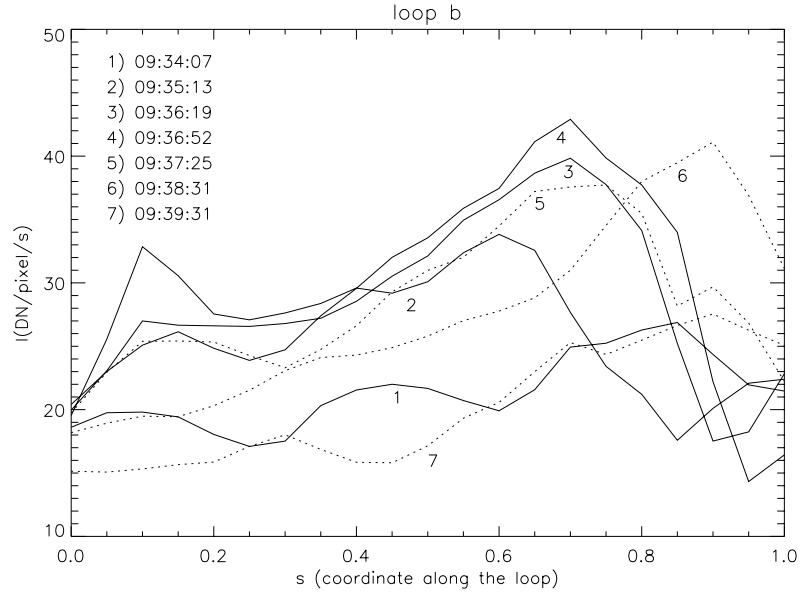

loop c

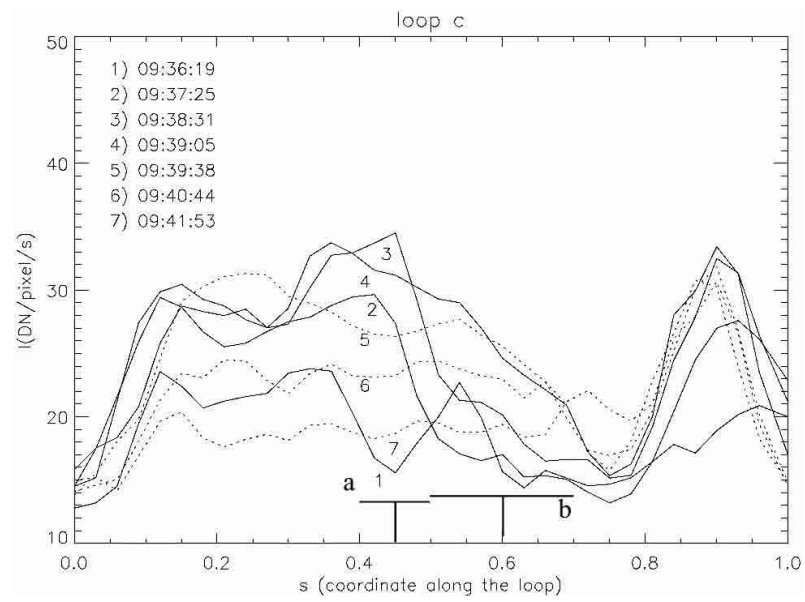

Fig. 9. Intensity $I$ (in DN/pixel/s) as a function of $s$, the coordinate along the loop at 7 different times for loops $a, b$ and $c$, respectively. Curves 1-4 (solid line) refer to times before the $\bar{I}$ maximum (as deduced by Fig. 8a); curves 5-7 (dashed line) to times after the maximum. In the third graph (from the top) the intervals on the axis of the abscissae labelled (a) and (b) indicate the regions of contact between loop $c$ and loop $a$ and $b$ respectively.

indicates that both in the ascending and descending phase, there are two regions of maximum intensity: one, the most extended, coincides with the southern leg of loop $c$, the other is close to the northern footpoint. This graph indicates that the region of apparent contact between loops $c$ and $b$, even if showing a variable brightness in time, is always the faintest in the system.

On the basis of this analysis, we argue as follows: if the loops were only in an apparent contact (i.e. they were overlapping along the line of sight but were not in physical contact), they would have probably shown a more constant brightness during the considered time interval; the observed variations in brightness in the sites close to the apparent crossing region could mean that they were physically interacting, since the interaction could have caused variations in density or temperature and so lowered the intensity in the crossing region at $171 \AA$.

In order to investigate further this possibility, we have analyzed the magnetograms of AR 8421 taken at Mitaka National Astronomical Observatory of Japan and have deduced the field lines topology using both a potential field and a force-free field extrapolation.

Figures $10 \mathrm{a}$ and $10 \mathrm{~b}$ give the TRACE $171 \AA$ image of the flare No. 1 site and the BBSO $\mathrm{H}_{\alpha}$ image of AR 8421, respectively. In Fig. 10c we can see a map of the longitudinal magnetic field (where a continuous line means positive polarity and a dashed line means negative polarity) and of the azimuthal field (segments). Comparing this map with Fig. 10a, we conclude that flare No. 1 occurred in the region showing the highest degree of shearing (the azimuthal field is almost parallel to the magnetic neutral line) and therefore the sites where the magnetic field is very far from a potential field configuration. This magnetic field topology has been recognized as one of the most important precursors of flares (Hagyard et al. 1984).

Moreover, we show in Fig. 10d a potential field extrapolation, obtained by using the photospheric magnetic field values as boundary conditions (Sakurai 1981), where we can see loops crossing at right angles to the main polarity inversion line. Figures 10e (top view) and 10d (side view), showing a non linear force-free field extrapolation, reproduce more realistically the morphology of AR8421 observed in the $\mathrm{H}_{\alpha}$ line (see Fig. 10b). The values of $\alpha$ in the field lines involved in flare No. 1 and shown in bold in Fig. 10e are 0.3 (the northern) and -0.1 (the southern), respectively. Moreover, in the forcefree field extrapolation the region where flare No. 1 takes place is characterized by nearby field lines with different orientation, which cross each other. Therefore, the flare site is characterized by a magnetic field topology which is very favourable to field line reconnection.

Using the procedure described in Sect. 3, we have determined the physical parameters of these particular loops and have evaluated that, in a volume of $\sim 10^{24} \mathrm{~cm}^{-3}$, during the time interval 09:25 to 09:53 UT the electron density, pressure and thermal energy, show variations in the following ranges: $N_{\mathrm{e}} \sim 4.5-8.5 \times 10^{9}\left(\mathrm{~cm}^{-3}\right), P \sim 1.2-2.3\left(\right.$ dyne $\left.\mathrm{cm}^{-2}\right), E_{\mathrm{th}} \sim$ $8.3 \times 10^{23}-3.9 \times 10^{24}(\mathrm{erg})$.

These values would be characteristic of a small flare, but in soft X-rays flare No. 1 was classified as C5.1, indicating that it cannot be limited to this particular system of loops; rather they represent only part of a larger event.

In this context, we utilize all the information we have obtained from our analysis in an attempt to identify this flare in terms of the scenaria described in Sect. 4. Qualitative 


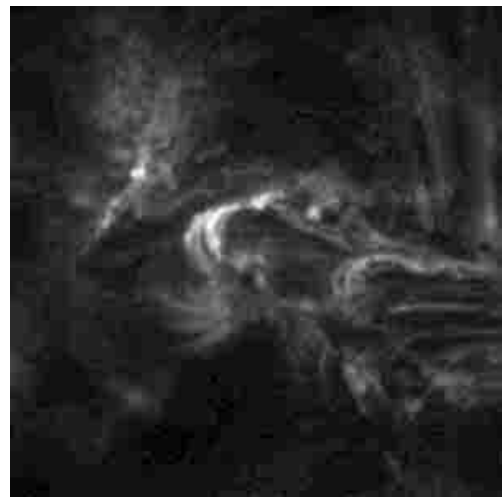

(a) $171 \AA$ 09:39:05 UT

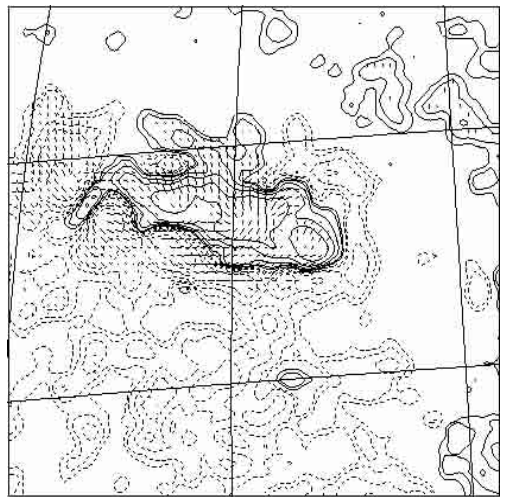

(c) longitudinal and azimutal field

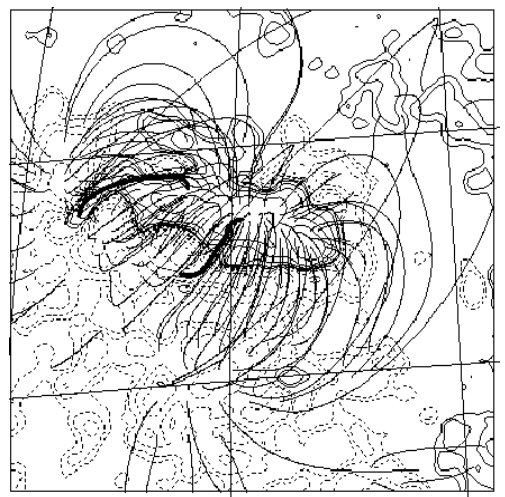

(e) force - free field (top view)

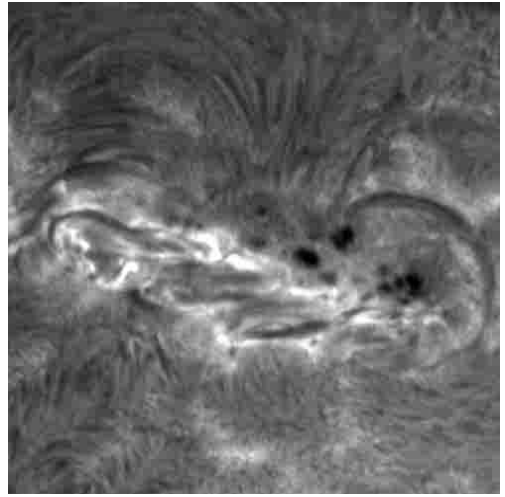

(b) $\mathrm{BBSO} \quad H_{\alpha} \quad 21: 49: 15 \mathrm{UT}$

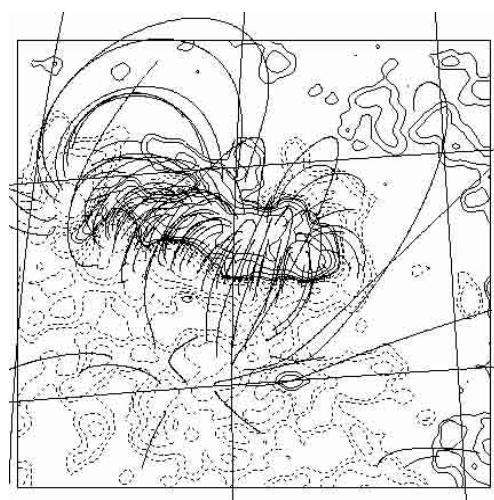

(d) potential field

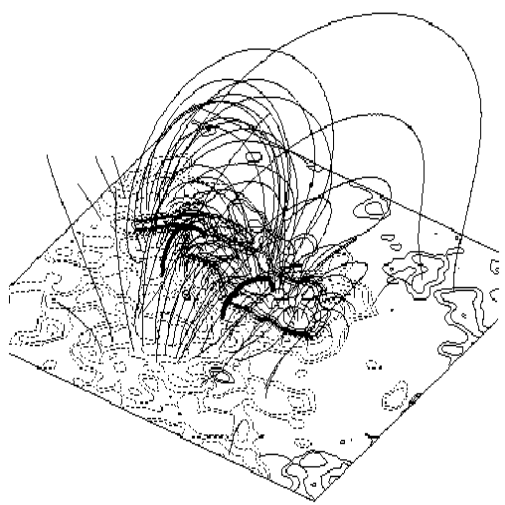

(f) force-free field (side view)

Fig. 10. a) $171 \AA$ image taken by TRACE on December 29 at 09:39:05 UT (fov $105000 \times 105000 \mathrm{~km}^{2}$ ); b) BBSO $\mathrm{H}_{\alpha}$ image of AR 8421 on December 29 at 21:49:15 UT; c) map of the longitudinal (continuous line indicates positive polarity, dashed line negative polarity) and azimuthal magnetic field (segments) observed at 01:38:25 at Mitaka National Astronomical Observatory of Japan; d) potential magnetic field extrapolation; e) force-free magnetic field extrapolation (top view), the field lines in bold indicate the locations of loops involved in flare No. 1; f) force-free magnetic field extrapolation (side view).

answers (when possible) to the questions $\left(Q_{1}, Q_{2}, \ldots\right.$ etc.) posed in Sect. 4 are as follows.

$Q_{1}$ : The emission comes mainly from a limited number of coronal loops which cross the main PIL, and we also observe brightness variations in patches of moss on both sides of the PIL, which are probably the footpoints of highertemperature loops (Martens et al. 2000).

$Q_{2}$ : The curves shown in Fig. 9 indicate that the intensity does not decrease along each loop simultaneously, which suggests that the loops are not cooling. Rather, we observe that the intensity increases first in certain locations and later the regions of maximum brightness change their position along loops $a$ and $b$, while in loop $c$ there is a continuous intensity increase at both sides of the apparent contact regions in the rise phase and a continuous intensity decrease during the main phase.

$Q_{3}$ : Loop $a$ shows an initial intensity increase in two locations, followed, after a time $\Delta t=66 \mathrm{~s}$ (calculated as the time 


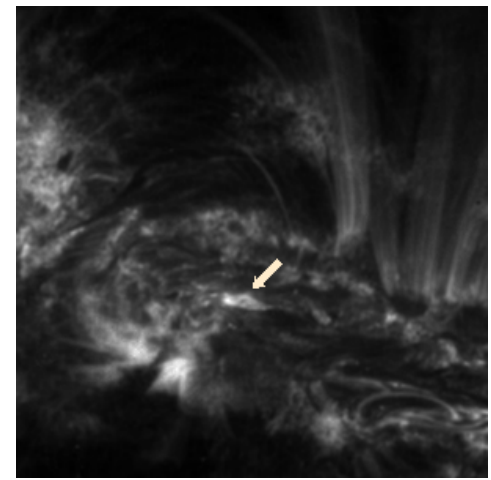

(a) 05:18:03 U.T.

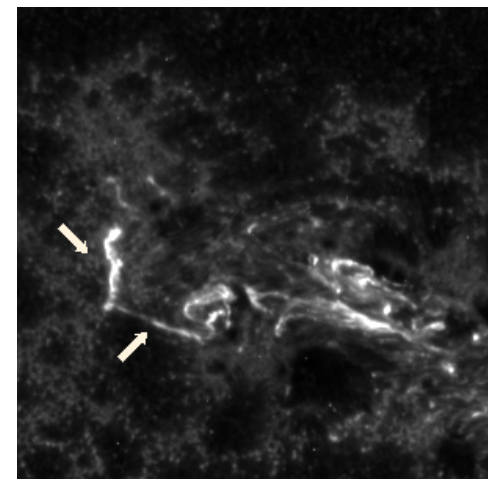

(c) $05: 36: 44$ U.T.

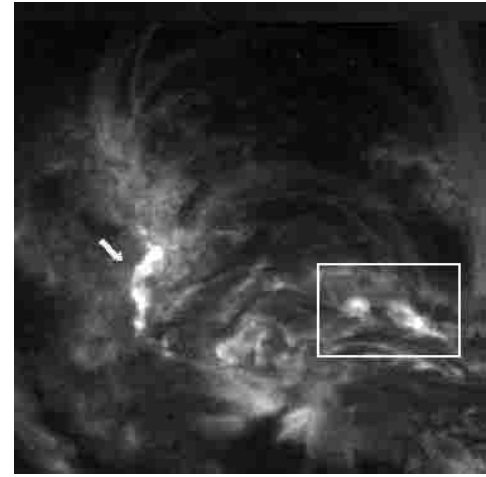

(b) 05:40:07 U.T.

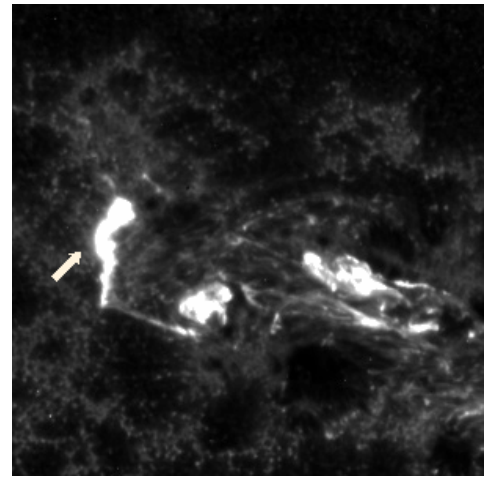

(d) $\quad$ 05:38:44 U.T.

Fig. 11. Sequence of images of the region shown in Fig. $5 b$ where flare No. $2-2^{\prime}$ occurred on December 30 . In frame a) we show a $171 \AA$ image, where the white arrow points to the site where the initial intensity enhancement took place during flare No. 2. In frame b) we show two patches of moss, indicated by an arrow and a box, respectively, where flare No. $2^{\prime}$ was observed. Frames c) and d) show $1600 \AA$ images of the same region: in c) the lower arrow shows an intensity increase along a structure connecting a central loop-like structure with the eastern moss (left arrow); in d) the two moss sites on both sides of the main PIL are shown.

difference between curves 2 and 3 of Fig. 9a), by a shift and simultaneous rise in intensity towards the apparent contact region. Loop $b$ shows an increase of intensity in a certain location, followed by a decline there and a rise in intensity either side of it (compare curves 1, 2 and 3 in Fig. 9b). Loop $c$ shows a different behaviour: an intensity increase at both legs (see curves 1-3) which begins after $\sim 2$ min of the rise phase in loop $b$.

$Q_{4}$ : After the brightening of loops $a, b$ and $c$, other loops, connecting both sides of the PIL in the western part of AR 8421, show an intensity increase. These loops appear in the force-free field extrapolation shown in Figs. 10e, f, to belong to the same arcade bridging the main PIL.

$Q_{5}$ : Our analysis of loops $a, b$ and $c$ has indicated that loops $a$ and $b$ show intensity variations along their length which may be caused by a heating source at their crossing point and this is evidence in favour of the hypothesis of a looploop interaction with consequent reconnection.

Therefore the scenario which results from the analysis of this flare is in accordance with the two-ribbon flare model of Heyvaerts et al. (1977), characterized by new flux tubes emerging close to a magnetic arcade, although we do not have the observations required to identify the emerging loops. When these flux tubes interact with loops belonging to the already sheared, non-potential arcade, and reconnect with them, this process could give rise to an instability which involves other loops in the arcade, and producing a two ribbon flare.

\section{Flare No. 2, observed on December 30 at 05:46 UT, M1.0}

On December 30, between 05:10 and 06:00 UT two flares (No. 2 and $2^{\prime}$ in our classification, see Table 2) were registered by GOES in AR 8421 (see the map in Fig. 5b). Despite the fact that GOES registered two distinct flares, we could determine, from the analysis of $1600 \AA$ images, that they were related and constituted two different parts of a single event.

From the analysis of $171 \AA$ images, we notice, at 05:15:18 UT, the appearance of many loops whose apparent contact points show a faint emission in the area of coordinates $27 \mathrm{~N} 01 \mathrm{~W}$ (coincident with the coordinates given by the NOAA report for flare No. 2). At 05:18:03 UT we observe an intensity increase in the region indicated by the arrow in Fig. 11a and later a gradual brightening of the surrounding structures, until 05:20:49 UT, when we observe the formation of two main parallel structures characterized by the presence of many bright knots.

In the region of coordinates $28 \mathrm{~N} 03 \mathrm{E}$, characterized by the presence of the moss and indicated by a white arrow in 


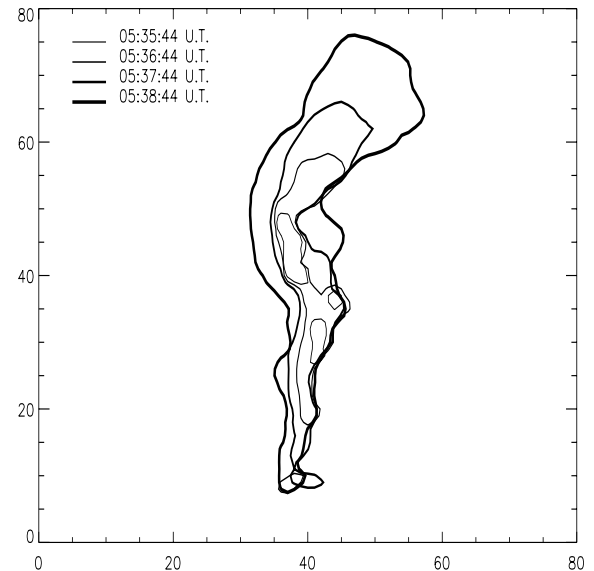

(a)

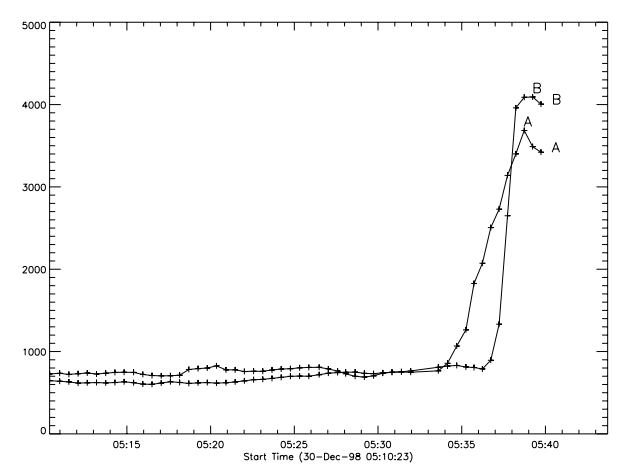

(c)

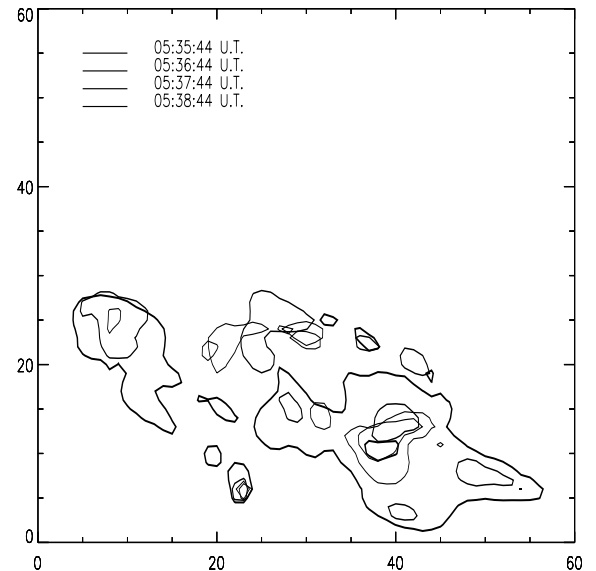

(b)

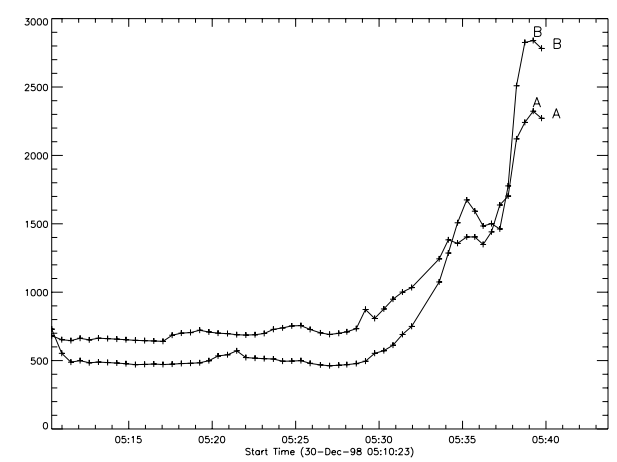

(d)

Fig. 12. a) Isocontours of intensity levels in the eastern moss at four different times. The numbers on the axes indicate the number of pixels from an arbitrary origin. b) Isocontours of intensity levels in the western moss at four different times. In graph c) we report the $1600 \AA$ intensity (in $\mathrm{DN} \mathrm{s}^{-1}$ ) as a function of time for the southern (from 0 to 40 pixels on the $y$-axis of frame a), curve A) and northern (from 40 to 80 pixels on the $y$-axis of frame a), curve B) parts of the eastern moss, respectively. In graph d) we show, with the same criterion used in graph c), the behaviour of $1600 \AA$ A intensity for the western moss.

Fig. 11b, which shows the image taken at 05:40:07 UT, there is a sudden increase in the emission coming mainly from two blobs of plasma inside the moss. These blobs increase their intensity and area until 05:46:20 UT, when the flare peak of flare No. $2^{\prime}$ was registered by GOES. Then, the emission in the moss slowly decreases. Moreover, during this time interval, we observe an intensity enhancement in two other patches of moss (bounded by a box in Fig. 11b), localized on the other side of the main PIL with respect to the largest moss patch.

The analysis of the set of images taken at $1600 \AA$ has given further details on this flare: initially we notice an intensity enhancement of loop-like structures in a region spatially coincident with that indicated by the arrow in Fig. 11a). Next, we observe an intensity enhancement in a structure which connects the site No. 2 with the site No. 2' (lower arrow in Fig. 11c showing the image taken at 05:36:44). Figure 11d shows the flare sites at 05:38:44 UT.

Figures $12 \mathrm{a}, \mathrm{b}$, display the isocontours of intensity levels in the eastern and western moss, respectively, at four different times, between 05:35:44 and 05:38:44 UT, as obtained from the analysis of $1600 \AA$ images.

Figures $12 \mathrm{c}$, d show the $1600 \AA$ intensity (in DN/s) in the southern (curve A) and northern (curve B) part of each moss, as a function of time. We can notice the abrupt intensity rising between 05:34 and 05:39 UT, due to the moss ignition. Moreover, comparing the curves referring to the southern and the northern part of the eastern moss, it is possible to note a time delay of the order of $\sim 2 \mathrm{~min}$ in the sudden intensity rise between the two parts of this moss. In contrast, the other moss is characterized by an intensity increase occurring simultaneously in the southern and the northern area.

In Fig. 13a we show the $171 \AA$ image of flare No. 2-2', in which the two moss sites are evidenced by an arrow and a box, respectively. In Fig. 13c a map of the longitudinal and azimuthal magnetic field of AR 8421 on December 30 is reported. In Figs. 13d, e, f we show the potential field, the forcefree field top view and side view extrapolations, respectively. Comparing the $171 \AA$ image with the force-free field extrapolations, it appears evident that the moss sites showing the intensity enhancements, are connected by large scale loops (field lines in bold in Fig. 13e, characterized by $\alpha=0.6$ ) and, taking into account that the moss represents the coronal feet of a hotter arcade of loops (Martens et al. 1999), we may presume that the intensity increase in the moss both sides of the magnetic inversion line could be caused by the same mechanism which 


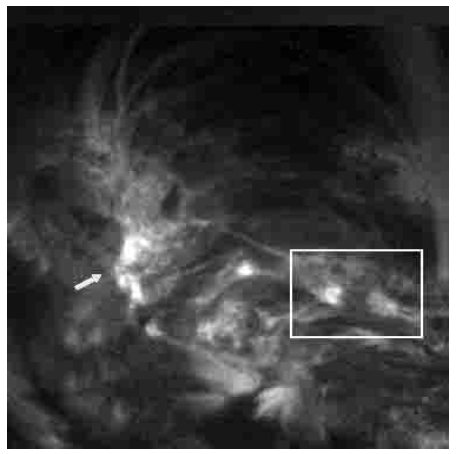

(a) $171 \AA 05: 40: 07 \mathrm{U} \mathrm{T}$

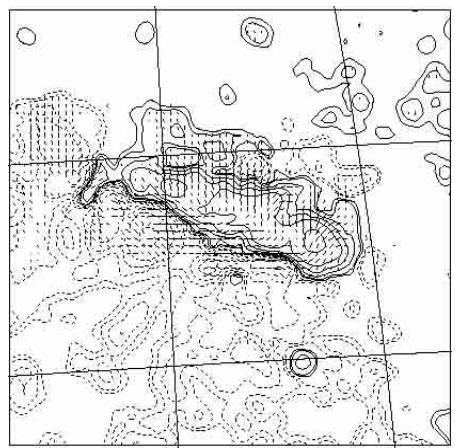

(c) longitudinal and azimuthal field

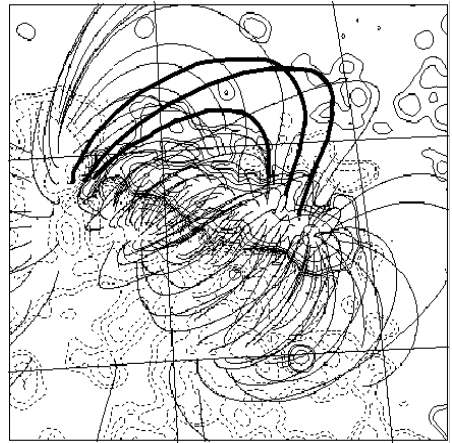

(e) force - free field (top view)

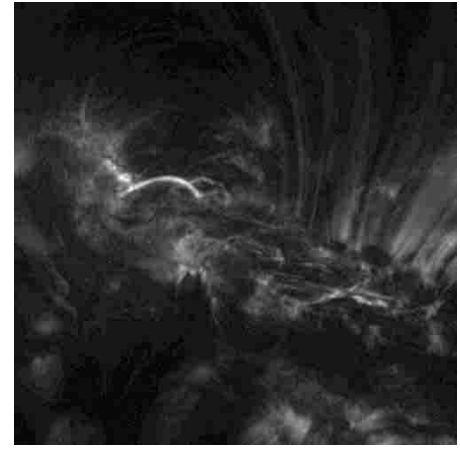

(b) $171 \AA 18: 21: 51 \mathrm{U} \mathrm{T}$

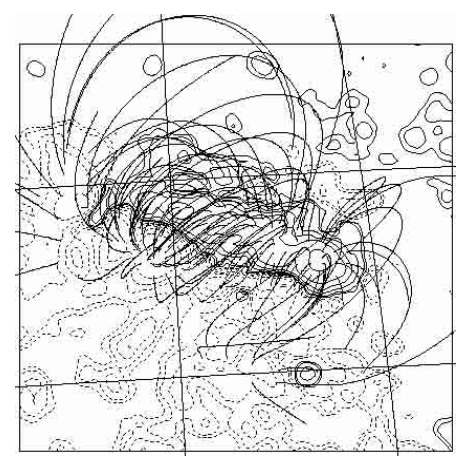

(d) potential field

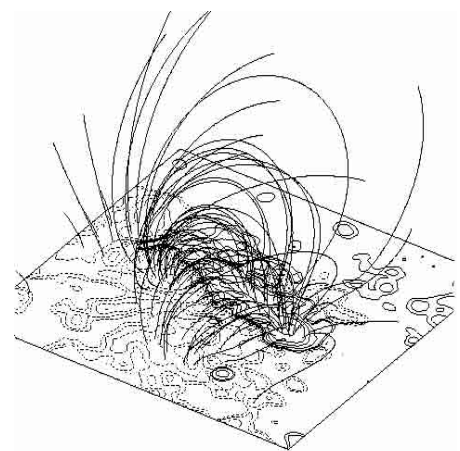

(f) force - free field (side view)

Fig. 13. a) $171 \AA$ A image taken by TRACE at 05:40:07 UT (fov $105000 \times 105000 \mathrm{~km}^{2}$ ), showing the moss brightening during flare No. 2-2'; b) $171 \AA$ image taken by TRACE at 18:21:51 UT (fov $175000 \times 175000 \mathrm{~km}^{2}$ ), showing AR 8421 during flare No. 3; c) map of the longitudinal (continuous line indicates positive polarity, dotted line negative polarity) and azimuthal (arrows) magnetic field observed at 02:25:39 at Mitaka Astronomical Observatory of Japan; c) potential magnetic field extrapolation; d) force-free magnetic field extrapolation (top view), the field lines in bold indicate the loops connecting the two moss regions involved in flare No. $2-2^{\prime}$; e) force-free magnetic field extrapolation (side view).

causes flare ribbons at optical wavelenghts, i.e. heating due to particle acceleration from a higher reconnection site.

In this regard, it is interesting to note that, considering the flare start time given by GOES (05:26 UT), and the moss light curves shown in Figs. 12c, d, we deduce that the time delay between the first flare manifestation in soft X-ray and the moss ignition in the EUV range is $\sim 9 \mathrm{~min}$.

Physical parameters such as electron density, pressure and thermal energy have been calculated for the two moss regions indicated in Fig. 11c via the procedure described in Sect. 3. They show a variability range of $1.7-4.8 \times 10^{9} \mathrm{~cm}^{-3}$,
$0.5-1.7$ dyne $\mathrm{cm}^{-2}$ and $1.3-3.7 \times 10^{25} \mathrm{erg}$, respectively, during the time interval 05:10 to 05:49 UT.

In the following we try to give answers to the questions posed in Sect. 4.

$Q_{1}$ : The emission comes initially from loop-like structures having dimensions at the telescope resolution limit and later from patches of moss on both sides of the polarity inversion line, which have been recognized as representing the coronal feet of hotter arcades of loops (Martens et al. 1999). 


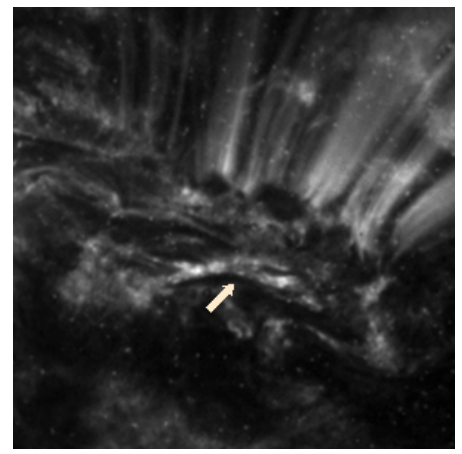

(a) 18:04:38 U.T.

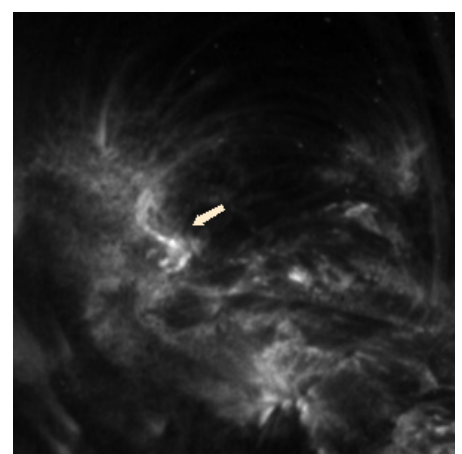

(c) 18:08:42 U.T

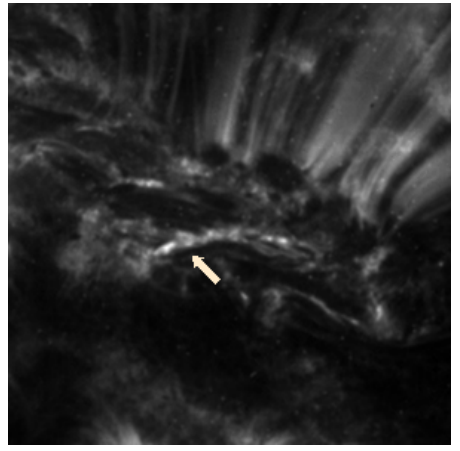

(b) 18:13:04 U.T.

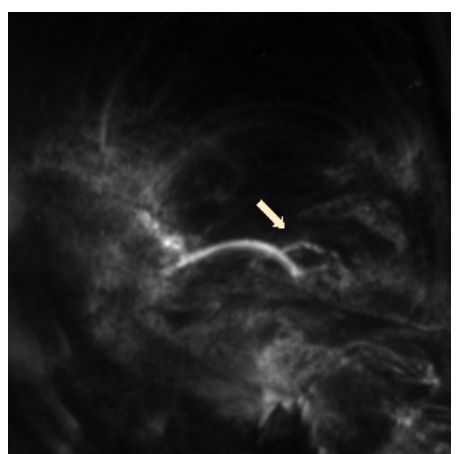

(d) 18:21:51 U.T.

Fig. 14. a), b): $171 \AA$ images of the region bounded by box No. 3a in Fig. 5c where two bundles of loops showed intensity variations on December, 30, at 18:10 UT (flare peak time). c), d): $171 \AA$ images of the region bounded by box No. $3 b$ in Fig. $5 \mathrm{c}$ where an intense moss brightening occurred during flare No. 3. In each frame the white arrow points to the site where major changes took place.

$Q_{2}$ : During the rise phase of flare No. 2 we observe loop-like structures, but their dimensions and their extremely rapid changes in shape and emission, do not allow us to determine intensity, density and emission measure variations in time and in space, so it is not possible to establish whether these structures are cooling or heating.

$Q_{3}$ : The flare morphology does not allow us to single out a heating source.

$Q_{4}$ : We observe intensity enhancements in the moss on both sides of the PIL. This may be a consequence of coronal loop reconnection and particle acceleration.

$Q_{5}$ : The loop-like structures involved in this event are such that no consideration is possible on their possible interaction.

Therefore, taking into account the evolution of the phenomena during this flare, the fact that initially the intensity increase takes place in the inner loops and later at the footpoints of larger and outer loops, this event is a two ribbon flare, characterized by an arcade of loops showing variable brightness and located at rising heights as time proceeds and the overlying reconnection points rise.

\section{Flare No. 3, observed on December 30 at 18:10 UT, C1.7}

During the time interval 18:04-18:30 UT, in AR 8421 we observe two regions, characterized by structures which evolve rapidly (see the $171 \AA$ map in Fig. 5c, showing the situation at 18:10:22 UT). The first region, situated at $11 \mathrm{~W} 25 \mathrm{~N}$ (not coincident with the coordinates given in the NOAA report), is characterized by two bundles of loops (Fig. 5c, box 3a), which show variable brightness and bright knots moving along their legs. The second region of interest, situated at 04W 30N (also not coincident with the coordinates given in the NOAA report) (Fig. 5c, box 3b), is characterized by rapidly evolving phenomena in the moss.

Let us examine separately the two regions.

\section{- Region A: Interacting loops}

Initially, there are two main bundles of loops (Fig. 14a) which show an X-type configuration (Shimizu et al. 1994). During the rise phase, one of the two bundles becomes brighter, while the other decreases its intensity. During this phase we observe, in the brighter loop, a motion of bright knots from the top to the footpoints. At 18:10:22 UT there is an increase in intensity of the eastern leg with the appearance of a bright knot which, in the following frames, seems to split in two knots of smaller dimensions and lower brightness. They move in opposite directions (with an average velocity of $\sim 30 \mathrm{~km} \mathrm{~s}^{-1}$ ) and show a decreasing emission with time (Fig. 14b). In the main phase (between 18:16:20 and 18:29:37 UT) we observe the formation of thin and faint loops surrounding the main structure.

\section{- Region B: The moss}

The region indicated in the box $3 b$ in Fig. $5 c$ is characterized by the presence of moss in which an S-shaped 
structure increases its brightness and from which a loop appears during the main phase ( 18:17 UT) (Figs. 14c, d). The analysis of the images indicates that inside the $S$ shaped structure there is a strong brightness variation, especially in the eastern part, where the left loop footpoint is anchored. Moreover, a continuous plasma motion is observed in the loop from the eastern to the western footpoint. During this motion we observe, close to the western footpoint, the appearance of a fainter loop.

Figure 15 displays a $1600 \AA$ image of the region shown in Figs. 14c, d; the dotted white line gives the location of the loop which appears at 18:17 UT. In the same figure we show the $1600 \AA$ isocontours (continous grey line) at five different times (between 18:13 and 18:20 UT) and hard Xray isocontours (continuous white line), obtained by HXT instrument of YOHKOH. Comparing Fig. 15 with Figs. 13e and 13f, we may infer that the hard X-ray isocontours map the sites of the tops of large-scale loops, while the $1600 \AA$ isocontours indicate the sites of loop footpoints. Therefore, if the hard X-ray isocontours indicate the sites where reconnection takes place, the $1600 \AA$ isocontours map the sites heated as a consequence of plasma flows and particle acceleration from the reconnection site. In this view, the loop which appears during the main phase is probably filled with chromospheric plasma evaporated as a consequence of particle bombardment from the reconnection site (Antiochos et al. 2000). Considering that the loop length is $\sim 24000 \mathrm{~km}$, and that the time interval between the image where the loop is not visible and the one where the loop is clearly visible, is $\sim 4 \mathrm{~min}$, if we assume that the loop is filled with plasma evaporated from the chromosphere, we obtain a value of $\sim 100 \mathrm{~km} \mathrm{~s}^{-1}$ for the evaporation velocity.

Therefore, the answers to the questions reported in Sect. 4 are:

$Q_{1}$ : We have observed two strongly emitting regions during flare No. 3; the first one was characterized by two bundles of loops showing a variable brightness and knots which moved at an average velocity of $\sim 30 \mathrm{~km} \mathrm{~s}^{-1}$, the second by patches of moss where a loop, which appears during the main phase, is anchored.

$Q_{2}$ : We have not observed an intensity decline in unison in the loops involved in this event, and so there is not signature of a uniform cooling, but initially the intensity increased in a narrow region coincident with the apparent crossing point between the loops.

$Q_{3}$ : The region of maximum brightness in the loops was located at their apparent crossing point during the rise phase; later, bright knots diverging from this site were observed. We stress however that during this flare a sudden intensity increase was observed in the moss, in a region distant $\sim 80000 \mathrm{~km}$ from the loops. It seems very likely that in this region chromospheric evaporation took place, which was observed in a loop, anchored in the moss, and suddenly filled with plasma.

$Q_{4}$ : The analysis of this event indicates that the loops we have observed are two-ribbon flare loops belonging to a magnetic arcade of greater dimensions.

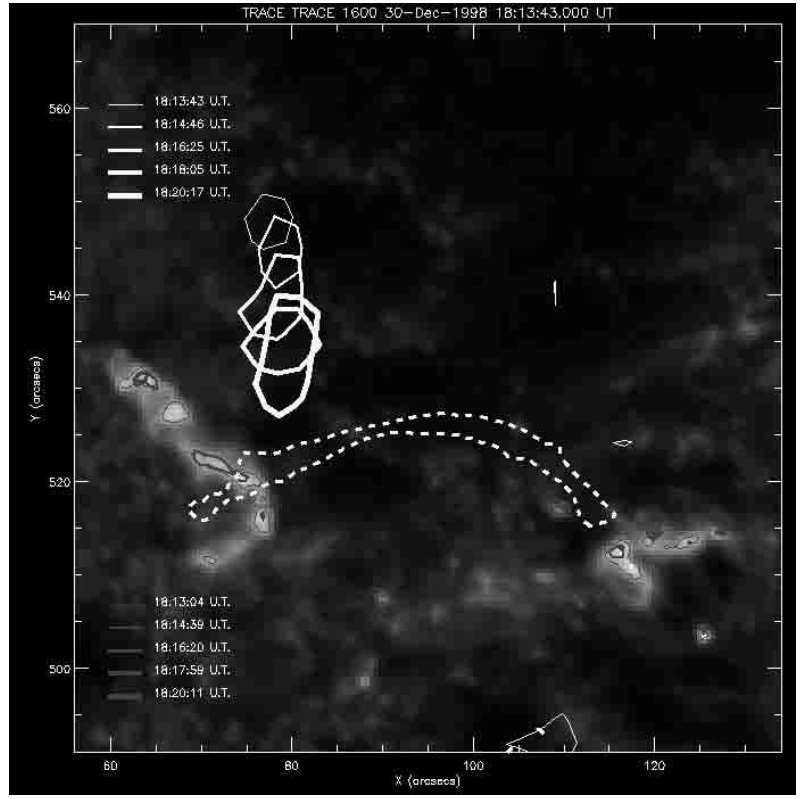

Fig. 15. $1600 \AA \AA$ image of the region shown in Figs. 14c, d. Grey lines indicate $1600 \AA$ isocontours at five different times. White dotted lines indicate the location of the loop that appeared during the main phase. White continuous lines indicate hard X-ray isocontours.

$Q_{5}$ : Observations suggest that there is a probable heating source at the crossing point of the loops.

This event represents observational support for the two-ribbon flare model based on magnetic reconnection as it showed several phenomena described in this model, that is: inner interacting loops, hard X-ray enhancements at large-scale loop tops, moss brightenings at loop footpoints and the sudden appearance of a loop probably filled by chromospheric evaporation.

\section{Discussion and conclusions}

We have examined images of AR 8421 at several wavelengths during the evolution of 3 flares characterized by energies, in the soft X-ray range, between $1.6 \times 10^{-3} \mathrm{erg} \mathrm{cm}^{-2} \mathrm{~s}^{-1}$ and $10^{-2} \mathrm{erg} \mathrm{cm}^{-2} \mathrm{~s}^{-1}$ (see Table 2). Let us briefly summarize the results obtained by the analysis of these flares.

During flare No. 1 we have observed emission in the form of a limited number of coronal loops having their footpoints anchored on both sides of the main polarity inversion line of AR 8421, together with emission coming from patches of moss situated on both sides of the PIL, but distant from the loops. We have established that there is a system of at least three loops showing, especially during the pre-flare phase, intensity variations in time and along their length which may be interpreted as due to an interaction between new flux tubes emerging close to an already sheared, non-potential arcade. As a consequence of this interaction, the new flux tubes and the nearer loops of the arcade reconnect, giving rise to a two ribbon flare. In this scenario, the increased emission in the moss is likely to be due to the same mechanism which causes $\mathrm{H}_{\alpha}$ ribbons, that is, heating by plasma flows from a higher reconnection site. 
Flares No. 2-2': we have initially examined this flare as two distinct events, following the indications given by the NOAA report, but a careful analysis of images taken at 171 and $1600 \AA$ has shown that they actually are two distinct manifestations of one large event. During the pre-flare phase we observed loop-like structures having dimensions at the angular resolution limit, which increased their emission.

The images at $1600 \AA$ showed a structure connecting the site of increased emission to the moss, where a sudden intensity enhancement took place; considering that the moss areas were connected by large scale loops, as confirmed by force-free field extrapolations, this intensity enhancement may be due to the same mechanism which causes flare ribbons at optical wavelengths, and is consistent with the two-ribbon flare reconnection model, characterized by heating of larger and larger loops occurring while the reconnection point rises in the solar atmosphere.

Flare No. 3: during this flare we observed two main emitting regions. The first was characterized by bundles of loops showing variable brightness and motion of bright knots, the second by brightening in the moss where a loop, which suddenly appeared during the main phase, was anchored. These observations may be interpreted in terms of the model of a two-ribbon flare triggered by magnetic reconnection at coronal levels: the hard X-ray enhancements and the moss brightening indicate the sites involved in the magnetic reconnection process. Moreover, as suggested by many authors, one of the consequences of magnetic reconnection and consequent particle bombardment to the lower atmosphere, is the chromospheric evaporation, characterized by upward motion of plasma toward the corona. Neupert (1968) has stressed that indirect evidence of chromospheric evaporation is that the observed soft $\mathrm{X}$-ray loops are filled with denser plasma than the surrounding corona, which may come from the chromosphere as a consequence of evaporation (Antiochos et al. 2000).

The velocity of the evaporation flow is a few hundred kilometers per second as measured by Doppler blueshifted lines during the rise phase of a flare (Feldman et al. 1980; Antonucci et al. 1982), but only $\sim 40 \mathrm{~km} \mathrm{~s}^{-1}$ in the magnetohydrodynamic simulation of a flare with chromospheric evaporation based on magnetic reconnection (Yokoyama \& Shibata 2001). In our analysis, we have obtained a value for the velocity of evaporation of $\sim 100 \mathrm{~km} \mathrm{~s}^{-1}$, which is consistent with previous observations.

These observations therefore support the interpretation of flares based on magnetic reconnection: when loops interact, in the contact region a current sheet forms; when the reconnection process takes place, accelerated particles are injected towards the loop footpoints and chromospheric material evaporates, filling the loops and increasing their luminosity.

As far as the observed brightness variations in the moss are concerned, we recall that Berger et al. (1999b) stated that moss is found only over magnetic plage areas that have associated $3-5 \times 10^{6} \mathrm{~K}$ coronal loops, as seen in Yohkoh/SXT images and this has led to the conclusion that moss is caused by thermal conduction from hot coronal loops overlying the plage. The high-pressure and temperature in the associated loops squeezes the emission at $10^{6} \mathrm{~K}$ into the loop legs and/or footpoints.
Theoretical models show that the association with high pressure and high temperature loops is a necessary condition for conductively heated moss (Peres et al. 1994). Martens et al. (2000) demonstrate that moss properties such as height and emission measure can only be explained by emission from a classical upper transition region below high-pressure hot loops and not by low-lying million-degree loops. Moreover, at the maximum spatial and temporal resolution of the TRACE instrument, moss does not appear to consist of small EUV loops. The intrinsic brightness of individual moss elements varies gradually with some elements appearing to fade in or out of existence on time scales of about $1 \mathrm{~min}$, and with no evidence of impulsive nano or microflaring as a major energy source for the moss. Flares observed in the moss by other authors (Berger et al. 1999b) indicate that moss is seen to form and spread behind post-flare loop arcade fronts that move outward from flare sites. The fronts correspond to the $\mathrm{H}_{\alpha}$ ribbons. The moss forming behind these fronts sometimes fades rapidly on time scales of $10 \mathrm{~min}$.

On the other hand, observations of chromospheric evaporation indicate the existence of a temporal correlation between hard X-ray bursts and the intensity of blue-shifted line emission. This suggests a strong relationship between evaporation and energy release because nonthermal electrons that are formed during the energy release process (possible reconnection) bombard the chromosphere and compress plasma there to drive the evaporation flow. Another evidence supporting the evaporation model is that the blueshifted soft X-ray emission comes from the footpoints of a soft $\mathrm{X}$-ray loop and that it is cospatial with the redshifted feature in $\mathrm{H}_{\alpha}$.

Our conclusions are: a) the characteristics of these flares are such that the analysis at $171 \AA$ allows us to study both loop interaction and moss brightenings, i.e. both the site where the triggering process may take place, and the atmospheric response; b) the flares are of two ribbon type, but show great complexity, with some of the loops changing their intensity due to loop interactions, while some are heating or filling with evaporated plasma and others are cooling or draining. Therefore we conclude that TRACE observations at $171 \AA$ may give the opportunity, under suitable circumstances, to observe in the same image the primary channels of energy release (enhanced UV emission in the reconnecting loops) and the secondary energy release (i.e. the hydrodynamical and radiative response of the solar atmosphere in the form of flare ribbons).

Before TRACE observations, we could observe these two distinct manifestations of the flare phenomenon only at different wavelength (and atmospheric) ranges: X-ray for the triggering site and optical $\left(\mathrm{H}_{\alpha}\right)$ for flare ribbons, which meant different instruments, different angular and temporal resolution and often the impossibility to evaluate the time interval between the primary and the secondary energy release.

However, it should be borne in mind that we are only observing loops of particular temperatures when many more loops of higher temperatures (invisible to TRACE) are also likely to be present. In future, it would clearly be beneficial to observe them too at the same time, but the present study over a limited temperature range has indicated the extreme complexity and dynamic nature of a flaring active region. 
Acknowledgements. The authors wish to thank the TRACE team for their open data policy and the free diffusion of Solar Software. Thanks are also due to T. Sakurai for providing the Software Package for the Computation of Solar Magnetic Fields.

\section{References}

Antiochos, S. K., De Luca, E. E., Golub, L., \& Mc Mullen, R. A. 2000, ApJ, 542, L151

Antonucci, E., Gabriel, A. H., Acton, L. W., et al. 1982, Sol. Phys., 78,107

Aschwanden, M. J., Schrijver, C. J., Alexander, D., \& Fletcher, L. 1999, ApJ, 520, 880

Aschwanden, M. J., Alexander, D., Hurlburt, N., et al. 2000a, ApJ, $531,1129 \mathrm{~A}$

Aschwanden, M. J., Tarbell, T. D., Nightingale, R. W., et al. 2000b, ApJ, 535, 1047

Berger, T. E., de Pontieu, B., Schrijver, C. J., \& Title, A. M. 1999a, ApJ, 519, L97

Berger, T. E., De Pontieu, B., Fletcher, L., et al. 1999b, Sol. Phys., 190,409

De Pontieu, B., Berger, T. E., Schrijver, \& Title, A. M. 1999, Sol. Phys., 190, 419

Davis, J. M., \& Krieger, A. S. 1982, Sol. Phys., 80, 295

Démoulin, P., Hénoux, J. C., Priest, E. R., \& Mandrini, C. H. 1996, A\&A, 308, 643

Feldman, U., Doschek, G. A., Kreplin, R. W., \& Mariska, J. T. 1980, ApJ, 241, 1175

Fletcher, L., \& De Pontieu, B. 1999, ApJ, 520, L135

Forbes, T. G., \& Priest, E. R. 1984, Sol. Phys., 94, 315

Freeland, S. L., \& Handy, B. N. 1998, Sol. Phys., 182, 497

Golub, L., \& 11 authors 1999, Phys. Plasmas, 6, 2205

Hagyard, M. J., Smith, Jr., J. B., Teuber, D., \& West, E. A. 1984, Sol. Phys., 91, 115

Hanaoka, Y. 1996, Sol. Phys., 165, 275-301

Handy, B. N., Acton, L. W., Kankelborg, C. C., et al. 1999, Sol. Phys., 187, 229

Heyvaerts, J., Priest, E. R., \& Rust, D. M. 1977, ApJ, 216, 123

Inda-Koide, M., Sakai, J.-I., Koide, S., et al. 1995, Publ. Astron. Soc. Japan, 47, 323-330

Krucker, S., \& Benz, A. O. 1998, ApJ, 501, L213

Lenz, D. D., De Luca, E. E., Golub, L., Rosner, R., \& Bookbinder, J. A. 1999, ApJ, 517, L155
Machado, M. E., Somov, B. B., Rovira, M. G., \& de Jager, C. 1983, Sol. Phys., 85, 157

Machado, M. E., Xiao, Y. C., Wu, S. T., Prokakis, Th., \& Dialetis, D. 1998a, ApJ, 326, 451

Machado, M. E., Moore, R. L., Hernandez, A. M., Rovira, M. G., Hagyard, M. J., \& Smith, Jr., J. B. 1998b, ApJ, 326, 425

Mandrini, C. H., Demoulin, P., Henoux, J. C., \& Machado, M. E. 1991, Astron. Astrophys., 250, 541

Martens, P. C. H., \& Kankelborg, C. C. 1999, AAS, 194, 7903N

Martens, P. C. H., Kankelborg, C. C., \& Berger, T. E. 2000, ApJ, 537, 471

Masuda, S., Kosugi, T., Hara, H., Tsuneta, S., \& Ogawara, Y. 1994, Nature V.371, No. 6497/Oct6, P. 495

Moore, R. L., \& Roumeliotis, G. 1992, in Eruptive Flares, ed. Z. Svestka, B. V. Jackson, \& M. E. Machado (New York: Springer), Lecture Notes in Physics, 399, 69

Neupert, W. M. 1968, ApJ, 153, L59

Parker, E. N. 1988, ApJ, 330, 474

Parnell, C. E., \& Jupp, P. E. 1999, ApJ, 529, 554

Peres, G., Reale, F., \& Golub, L. 1994, ApJ, 422, 412

Priest, E. R., \& Démoulin, P. 1995, J. Geophys. Res, 100, 23443

Priest, E. R., \& Forbes, T. G. 2000, Magnetic Reconnection (Cambridge University Press)

Priest, E. R., \& Forbes, T. G. 2002, A\&ARv, 10, 313

Sakurai, T. 1981, Sol. Phys., 69, 343

Schrijver, C. J., Title, A. M., Berger, T. E., et al. 1999, Sol. Phys., 187, 261

Shibata, K. 1997, Proc. Fifth SOHO Workshop. The Corona and Solar Wind near Minimum Activity, ESA SP-404

Shimizu, T., Tsuneta, S., Acton, L. W., et al. 1994, ApJ, 422, 906

Sturrock, P. A. 1980, Solar Flares - A Monograph from Skylab Solar Workshop II (Boulder: Colorado Associated University Press)

Tarbell, T. D., et al. 1994, Proc. 3d SOHO Workshop, Solar Dynamic Phenomena and Solar Wind Consequences (ESA SP-373; Paris: ESA), 375

Tsuneta, S., Hara, H., Shimizu, T., Acton, L. W., Strong, K. T. Hudson, H. S., \& Ogawara, Y. 1992, PASJ, 44(5), L63

Yokoyama, T., \& Shibata, K. 2001, ApJ, 549, 1160

Zuccarello, F., \& Romano, P. 1999, Proc. 9th European Meeting on Solar Physics, ESA SP-448; Florence, Italy, 907

Zuccarello, F., Contarino, L., \& Romano, P. 2001a, Proc. of the $2^{0}$ Convegno sulla Fisica Solare Italiana, Mem. Sait, 72(3), 579

Zuccarello, F., Contarino, L., \& Romano, P. 2001b, Sol. Phys., 199, 97 